\title{
Mechanistic Understanding of Size-Based Fibre Separation in Coiled Tubes
}

\author{
Jakob D. Redlinger-Pohn ${ }^{\mathrm{a}^{*}}$, Lukas A. Jagiello ${ }^{\mathrm{b}}$, Wolfgang Bauer ${ }^{\mathrm{b}}$, and Stefan Radl ${ }^{\mathrm{a}}$ \\ anstitute of Process and Particle Engineering, Graz University of Technology, Inffeldgasse \\ binstitute of Paper, Pulp and Fibre Technology, Graz University of Technology, Inffeldgasse \\ 13/III, 8010 Graz, Austria \\ *Corresponding author: E-mail: redlinger-pohn@tugraz.at; Tel.: +43 31687330421
}

Keywords: Particle Size Separation; Tube Flow Fractionator; Euler-Lagrange Simulation; Coiled Suspension Flow; Fibre Suspension Flow 


\begin{abstract}
Understanding separation of poly-disperse particle suspensions according to the particles size is of great importance to product quality. Previous experimental studies of suspension flow through coiled tubes report different results for spherical and elongated particles, e.g., larger and thus heavier elongated particles are faster than smaller ones.

We use Euler-Lagrange simulations, as well as experiments, to measure the residence time distribution of fibres with different size in coiled tubes with different curvatures. Fluid flow through the coiled tubes was simulated as toroidal flow, i.e., the pitch of the tube was neglected. Fibres are one-way coupled to the fluid, and their movement in the cross section, as well as their orientation is predicted based on the assumption of an infinitely dilute suspension.

We find that in coiled, dilute suspension flow of fibres the ratio of particle settling velocity to the secondary flow speed determines the fibre motion in the tube cross section. For low Reynolds number and thus larger effect of gravitation, fibres are found to concentrate in distinct orbits. Long fibres form flocs propagating through the torus whilst small fibres are well mixed and thus retained in the tube. We found that fibre-fibre interaction and the formation of flocs and not fibre-fluid interaction is key to the size based separation.
\end{abstract}




\section{Introduction}

Two-phase solid/liquid suspension flow in tubes and pipes is ubiquitous in the producing industry, including (i) pulp and paper manufacturing (Krogerus et al., 2003; Lundell et al., 2011), (ii) chemical and pharmaceutical industry (Eder et al., 2010), or (iii) recycling of resources (Carissimi and Rubio, 2005; Körkkö et al., 2008; Laitinen et al., 2008). A situation of high relevance is the flow through coiled and bent pipe configurations. Centrifugal forces acting on the fluid lead to (i) a deflection of the velocity maximum towards the outer bend, and (ii) a pressure difference between the inner and outer bend. The latter induces a secondary motion commonly known as Dean flow (Dean, 1928, 1927; Naphon and Wongwises, 2006; Vashisth et al., 2008). This secondary motion increases cross-sectional mixing, and hence reduces axial dispersion of suspended particles. Because the degree of mixedness of particles defines (i) product quality, or (ii) the capability to focus, align or even separate suspended particles (Di Carlo, 2009; Martel and Toner, 2013), a profound understanding of coiled suspension flow is essential. The current paper focuses on the exact mechanism behind the separation of suspended elongated particles, more specifically of fibres. Specifically, we are interested in how the fibre size, shape, and concentration affects the separation process in a coiled tube. Clearly, in case of a pipe with no lateral exit, separation of particles can be only realized in case the residence time of the particles is different from each other. Hence, there is a natural interest in the residence time of suspended particles in coiled tubes.

The influence of curvature and flow conditions on the particle residence time are well investigated for suspensions consisting of spherical particles (Koutsky and Adler, 1964; Palazoglu and Sandeep, 2004; Tiwari et al., 2006). For system involving non-spherical, elongated particles (e.g., fibres) additional modes of particle motion, such as flipping and tumbling, have to be taken into account (Jeffery, 1922; Rosén et al., 2014). Currently, studies on the residence time distribution (RTD) of elongated particles are limited to experimental results (Krogerus et al., 2003; Laitinen et al., 2011, 2006). Findings from literature are that the particle residence time decreases with fibre length. However, these previous studies lack a mechanistic description of the separation process: the current understanding is based on observations from fibres and particles separating in slug tube flow (Johansson et al., 1970; Olgard, 1970). 


\subsection{Objectives}

It is the purpose of this paper to provide a mechanistic explanation for size-based separation of fibres in a coiled pipe configuration. The focus is on intermediate Reynolds numbers which correlates with typical operational settings (Laitinen et al., 2006). In this range of Reynolds numbers we deal with steady-state, or oscillating pipe flow in toroidal configurations. We perform experiments to verify the influence of the Reynolds number and the concentration of the fibre pulp suspension on the separation process. The motion on dilute fibre suspension within toroidal fluid flow is then simulated with a recently developed open-source CFD-DEM code (CFDEM ${ }^{\circledR}$ project, DCS Computing GmbH (Goniva et al., 2012)). Figure 1 summarizes the simulation cases and experiments in the flow regime map proposed by Di Piazza and Ciofalo (Di Piazza and Ciofalo, 2011).

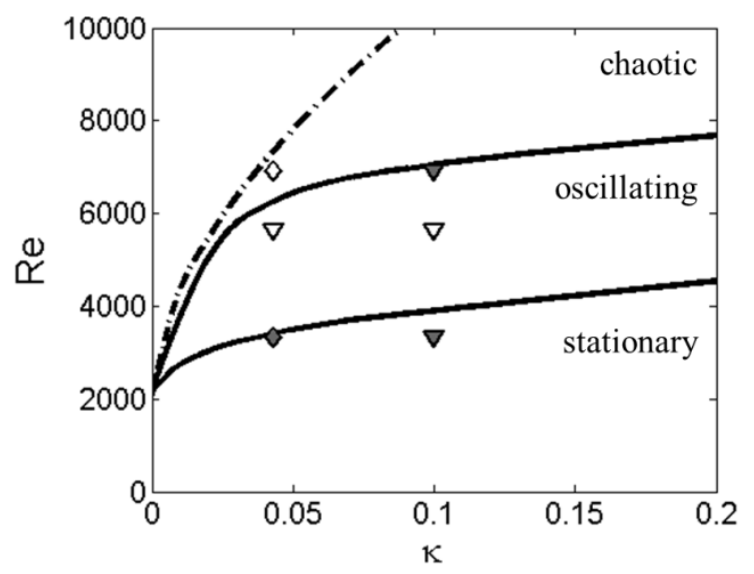

Figure 1: Regime map for flow through coiled pips (adapted from Di Piazza and Ciofalo (Di Piazza and Ciofalo, 2011). Simulation cases are denoted by triangles, where filled symbols denote two phase fibre/fluid (CFD-DEM) simulations. Diamonds represents cases were we both experiments and simulations were performed.

Specifically, the questions to be answered in the current paper are:

(1) How is the fibre position and trajectory affected by the fibre aspect ratio and pipe curvature?

(2) What is the fibre residence time distribution?

(3) What is the mechanism responsible for size-based fibre separation in a coiled tube?

\subsection{Outline}

The outline of the paper is as follows: 
In Section 2 we review recent studies on single-phase flow in coiled pipes, and fibre motion in suspension flow. This is to provide adequate background information on the most important flow features that affect particle trajectories.

In Section 3 we present supporting experiments using a fibre separation device, i.e., the tube flow fractionator (TFF). Specifically, experiments with sulfite pulp and mono-sized synthetic cellulose fibres were conducted at different settings of flow rate and fibre concentration. Also, the effect of fibre flocculation on the fibre movement in the channel is discussed.

In Section 4 we present the results of the numerical study. Fibres of different size are introduced into a toroidal tube flow. Fibre position in the pipe cross section, orientation and the resulting residence time of fibres in the pipe are discussed.

In Section 5 we link the simulation study and the experimental study and discuss the results.

In Section 6 we conclude the work and summarize our major findings.

The Appendix presents details related to the post-processing the numerical simulations. The electronical annex (see Electronic Annex in the online version of this article) presents results of our single-phase simulations of toroidal flow and details on the mesh generation, as well as the validation of DNS simulation results. 


\section{Theory on Toroidal Flow and the Motion of Fibres}

\subsection{Fluid Motion in Coiled Tubes}

In coiled fluid flow, a pressure difference between the outer and inner pipe bend, caused by centrifugal forces, leads to secondary motion within the cross section of the pipe. Dean (Dean, $1928,1927)$ showed that the curvature $\kappa=d / D$, the ratio of the pipe diameter $d$ to the coil diameter $D$ is of major importance to the secondary motion. Hence, the extent of the secondary motion is described by the Dean number $D a$, i.e., a Reynolds number $R e$ modified by the curvature:

$$
D a=\frac{u_{\mathrm{sec}} \cdot d}{v}=\operatorname{Re} \cdot \sqrt{\kappa}
$$

Here $v$ is the kinematic viscosity of the fluid and $u_{s e c}$ is the velocity of the secondary motion which scales with the square root of the curvature and the axial fluid motion, $u_{\mathrm{sec}}=u_{\text {bulk }} \sqrt{\kappa}$.

$D a$ characterizes the effect of inertial, viscous and centrifugal forces on the flow. The secondary motion is then often referred to as Dean flow, and the resulting vortices arising at the inner side of the bend as Dean vortices. $R e$ is based on the bulk velocity $u_{b u l k}$ and the pipe diameter $d$.

$$
\operatorname{Re}=\frac{u_{b u l k} \cdot d}{v}
$$

Recently, direct numerical simulation (DNS) of fluid flow through curved and helically curved pipes were performed to gain a better understanding of the complex phenomena in this flow system (Ciofalo et al., 2014; Di Liberto et al., 2013; Di Piazza and Ciofalo, 2011; Hüttl and Friedrich, 2001, 2000; Noorani et al., 2013). Specifically, it was found that due to centrifugal forces, the velocity maximum is deflected to the outer side of the bend, and the effect increases with the curvature. Consequently, the velocity gradient at the outer bend (i.e., near the outer wall) was observed to be high. Surprisingly, it was found that turbulence is suppressed, and hence the transition to turbulent flow occurred at higher $R e$. In an extensive numerical study, Di Piazza and Ciofalo (Di Piazza and Ciofalo, 2011) investigated the flow behavior in a toroidal domain for cases below the critical Reynolds number $R e_{c}$. They found that for increasing $R e$ 
the Dean vortices first start to oscillate. The oscillating behavior was also observed in an experiment using a mildly curved torus $(\kappa=0.049)$ (Kühnen et al., 2013). With further increase of $R e$, the flow becomes chaotic. As already mentioned, this however occurred at a critical Reynolds number larger than that in a straight pipe flow (Figure 1).

Studies comparing toroidal and helically coiled pipe flow showed, that torsion has only a weak effect on the axial main flow. It was found that peak turbulence is reduced at the outer side, and the pressure drop is unaffected (Ciofalo et al., 2014). However, torsion has an effect on the secondary motion. For high values of torsion the Dean vortex in the upper half of the pipe in relation to the direction of torsion increases, whilst the Dean vortex in the lower half of the pipe decreases (Hüttl and Friedrich, 2001, 2000). Again, for small torsion (ratio of pitch to coil radius smaller 1) the effect is negligible. A torus is hence a good and valid approximation of the industrially more commonly used situation of helically coiled pipes with small torsion.

\subsection{Effect of Secondary Motion on Suspension Flow}

For two-phase solid/liquid flow in coiled and bent pipes it is known that particles are additionally mixed in the pipe cross section due to the secondary motion. The mixing intensity increases with the curvature $\kappa$, i.e., with the intensity of the secondary motion. One effect is, that the particle residence time (RT) distribution is narrower compared to straight pipes (Koutsky and Adler, 1964; Palazoglu and Sandeep, 2004; Sandeep et al., 1997). Also, densitybased segregation was observed by the latter authors. However, particles with a density similar to the fluid are mixed intensively by the secondary motion. Additionally the residence time was observed to be lower than the hydraulic RT. In contrast, particles with higher density ratio accumulated close to the bottom wall at the inner side of the bend. Only for increased curvature the secondary motion was strong enough to prevent particles to accumulate (Tiwari et al., 2006).

\subsection{Motion of Elongated Particles and Fibres}

Due to their anisotropic shape, orientation and rotation of suspended fibres influences their trajectories when flowing through a pipe. For example, previous studies revealed that fibre-wall contact leads to a re-orientation of the fibres in the flow, and hydrodynamic interactions cause the fibre to rotate on a chaotic orbit (Jeffery, 1922; Lundell and Carlsson, 2010; Rosén et al., 2014). A primitive prototype for such an orbiting behavior in a simple shear flow is the so- 
called Jeffery orbit, which is characterized by the aspect-ratio-specific particle flipping frequency:

$$
\dot{\gamma}_{p}=\frac{\dot{\gamma}}{(\lambda+1 / \lambda)}=\frac{2 \pi}{\tau_{J O}} .
$$

Here $\dot{\gamma}$ is the fluid shear rate and $\lambda$ the fibre aspect ratio, i.e., the length of the major axis divided by that of the minor axis. $\tau_{J O}$ is the time to complete one Jeffery orbit. In straight channel and pipe flow at laminar conditions, fibres were found to align with the flow direction, and consequently fibres are pointing into flow direction with their major axis (Jianzhong et al., 2004; Krochak et al., 2009; Lin et al., 2003). Fibre density and aspect ratio had only a minor influence on fibre motion.

For turbulent flow conditions it was found that fibre orientation becomes more isotropic and fibres migrate to the viscous sub-layer. Fibre dynamics in turbulent channel flow recently became topic of DNS-based studies (Do-Quang et al., 2014; Marchioli and Soldati, 2013; Marchioli et al., 2010; Mortensen et al., 2008a, 2008b; Zhao and Van Wachem, 2013). Mortensen et al., as well as Marchioli et al. studied elongated particles, having different Stokes numbers. The effect of the fibre-fluid interaction on the fluid was neglected, i.e., one-way coupled simulations were performed. They found that fibres migrate towards the channel wall, into the viscous sub-layer. The drift velocity, the near-wall rotational motion, and the near-wall rotational fluctuations were affected by the particle aspect ratio. It was argued that particles possess a high enough inertia to be ejected of vortical structures, and accumulate in the viscous sub-layer. Fibres align according to the mean shear of the flow. In the pipe center, where only weak velocity gradients exist, fibres are more randomly distributed compared to the vicinity of the wall where larger shear forces are found. Fibres were found to accumulate in low-speed streaks caused by effects due to the fibres' inertia. Similar behavior was found experimentally (Kvick, 2014) for fibres in an open channel flow: (i) fibres clustered into streaks and (ii) fibre orientation in the near wall region was dominated by the fibre aspect ratio. Small fibres align in the vorticity direction, whilst longer fibres align into stream-wise direction. In summary, fibres showed a complex behavior, aligning or not depending on their density and size. 


\subsection{Crowding Number and the Formation of Fibre Flocs}

Accounting for fibre-fibre interaction in the suspension flow, it is known that fibres form coherent networks, typically referred to as fibre flocs, already at low mass concentration (Bennington et al., 1990; Chen et al., 2003; Cui and Grace, 2007; Derakhshandeh et al., 2011; Hourani, 1988). For suspension flow characterized by low shear rates, fibres contact, entangle and form flocs. Increasing the shear rate and overcoming the flocs's yield stress, the floc is ruptured and fibres move again freely in the suspension.

The formation of fibre flocs is highly dependent on the fibre concentration and changes with fibres aspect ratio and fibre flexibility. For fibres with a large aspect ratio, formation of fibre flocs occurs at lower concentration: for example, for flocs consisting of monodisperse polymer fibres it was found that yield stress increased strongly with the fibres' aspect ratio. For a polydisperse mixture of cellulose fibres, similar findings were reported, however, the effect was weaker. Simulating flexible fibres, Schmid and Klingenberg (Schmid and Klingenberg, 2000) closer investigated the effect of fibre stiffness and shape. They found that a too large flexibility of fibres will hinder the formation of flocs, since a certain amount of normal forces, compacting the fibres network and hence cause inter-fibre friction, is required (Switzer and Klingenberg, 2004).

A common measure in paper and pulp industry to describe the floc formation in dependence of the concentration and fibre length is the crowding number $N_{C W}$ (Kerekes, 2006). The crowding number describes the number of fibres expected in a sphere with a diameter equal to the fibre length:

$$
N_{C W}=5\left[\frac{k g}{m^{3}}\right] \cdot \frac{c_{m} L_{1}^{2}}{c s} .
$$

Here $c_{m}$ denotes the fibre mass concentration in percentage, $L_{1}$ the length-weighted average fibre length, and $c s$ is the fibre coarseness (weight per unit length of fibre). The crowding number was found to describe different fibre interaction regimes: $N_{C W}<1$ : chance of fibre-fibre collision, $1<N_{C W}<16$ : dilute fibre flow, $16<N_{C W}<60$ : fibre interaction but no immobilization, and $N_{C W}>60$ : formation of a strong coherent structure with immobilization of fibres (Kerekes, 2006). 


\section{Experiments}

As basis for a detailed numerical study, we first performed experiments on suspension flow of elongated particles in coiled tubes, namely in a Tube Flow Fractionator (TFF). The TFF is a long coiled pipe and was designed following literature suggestions (Jagiello, 2013; Krogerus et al., 2003; Laitinen et al., 2006). The TFF was tested with unrefined sulfite pulp (Sappi Gratkorn, Austria) against an empirical correlation from literature with respect to the mean residence time as a function of the fibre length (Laitinen et al., 2011). The subsequent residence time (RT) measurements in dependence on the Reynolds number $R e$ were performed using mono-disperse synthetic cellulose viscose fibres (Danufil ${ }^{\circledR}$, Kelheim Fibres GmbH, Germany).

\subsection{Materials and Methods}

The TFF is sketched in Figure 2. The key element is a $100 \mathrm{~m}$ long PVC pipe with smooth wall and an inner diameter of $0.016 \mathrm{~m}$ coiled around a drum with a diameter of $0.37 \mathrm{~m}$. Thus, the curvature resulted to $\kappa=0.043$. Note that the tube strength is increased with a tricot-polyester layer, however a perfect circular cross section cannot be guaranteed and a minor shape deviation (of the circular cross section) was observed. The feed section consisted of a water tank, a feed point with two 3-way valves, and two $90^{\circ}$ bends connected by a pipe of diameter $13.5 \mathrm{~mm}$.

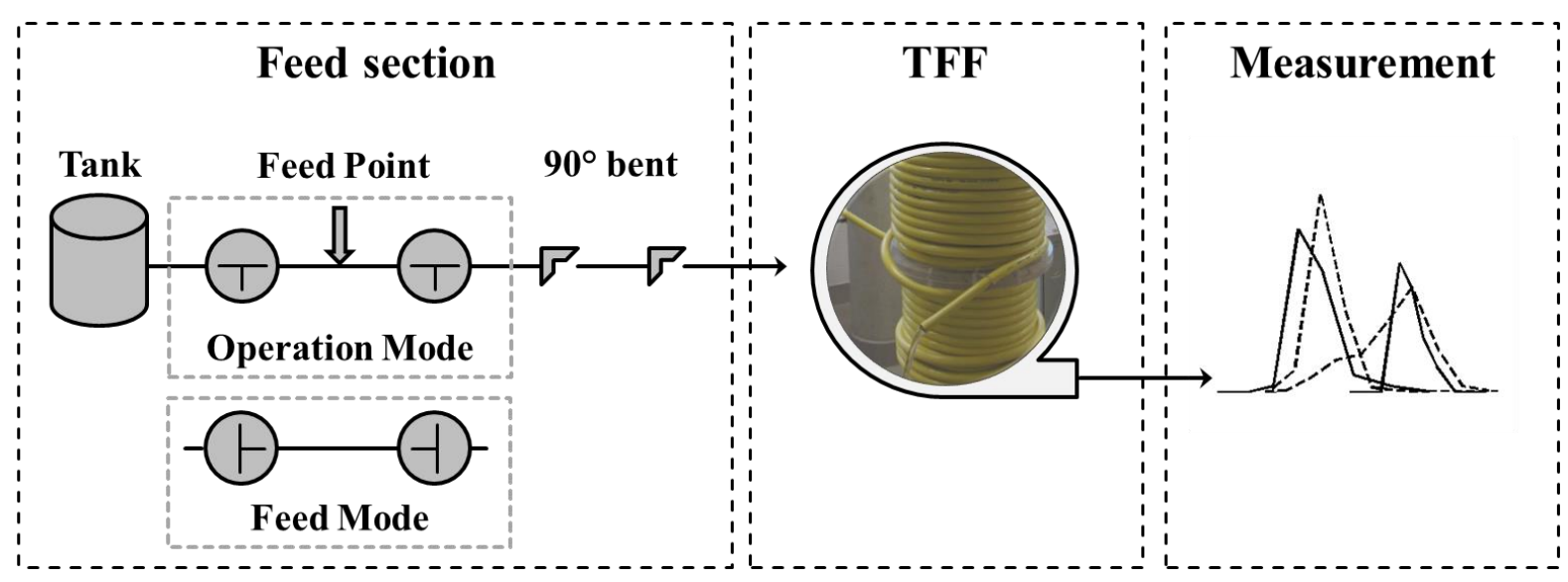

Figure 2: Experimental set-up of the tube flow fractionator. Feed section: tank, 3-way valve, feed point, $90^{\circ}$ bends. The position of the 3-way valve for operation and feeding is indicated. The tube flow fractionator consists of a $100 \mathrm{~m}$ pipe with an inner diameter $0.016 \mathrm{~m}$.

The 3-way valves were switched as indicated in Figure 2 for feeding the pulp suspension using a syringe. Water displaced from the pipe exited by the 3-way valves. By that, we could assure that no air bubbles are introduced when feeding the pulp suspension. The valves were switched 
into operation mode to start the experiment. Deionized water was supplied by the water tank. Fluid flow was ensured by a centrifugal pump and the flow rate was controlled by a PI (eControl 8611, Bürkert, Austria). The water temperature, ca. $22^{\circ} \mathrm{C}$, was uncontrolled but measured for one experiment where it rose from $22.2^{\circ} \mathrm{C}$ to $22.6^{\circ} \mathrm{C}$. The effects on viscosity and thus Reynolds number are thus negligible. The manufacturing and validation of the TFF were documented by Jagiello (Jagiello, 2013).

The fibre suspension was collected in bins at the exit of the TFF for different time instances. Sampling interval, which defines the time classes, was adjusted to the flow rate. However, the minimum time interval was $5 \mathrm{~s}$, since a manual sampling was performed. Fibre concentration per sample was measured gravimetrically for the RT distribution of mono-disperse suspensions of synthetic cellulose viscose fibres (CF) and the mass balance experiments. Fibres were collected off-line using a filter (MN 615, Macherey-Nagel, Germany) and dried at $105^{\circ} \mathrm{C}$. The mass of fibres per time instances was weighed. The fibre residence time distribution was calculated based on the mass distribution. For experiments with sulfite pulp (SP), as well as mixtures of $\mathrm{CF}$, the fibres length distribution per time instance was determined using the L\&W Fiber Tester Plus (Lorentzen \& Wettre, Sweden) according to the ISO 16065-2:2014 standard. The fibre residence time distribution was calculated based on the number distribution. All experiments were performed in duplicate.

Table 1 lists the physical data of CF. The crowding number for the mono-disperse fibre suspension was calculated for fibre mass concentration of $0.3 \%$ and $0.1 \%$ according to Eqn. 4 .

Table 1: Physical data of "Danufil ${ }^{\circledR}$ " synthetic cellulose fibres (Kelheim Fibres, Germany). Volumeweighted $\left(L_{3}, d_{3}\right)$ and length-weighted $\left(L_{1}, d_{1}\right)$ means are presented in the table. The crowding number is calculated according to Eqn. 4 for a fibre mass concentration of $0.3 \%$, and $0.1 \%$.

\begin{tabular}{lrcc}
\hline & & $\begin{array}{c}\text { nominal } \\
\text { length } \\
\text { 4 mm }\end{array}$ & $\begin{array}{c}\text { nominal } \\
\text { length } \\
\mathbf{0 . 3} \mathbf{~ m m}\end{array}$ \\
\hline Length $\left(L_{3}\right)$ & {$[\mathrm{mm}]$} & 3.90 & 0.31 \\
Width $\left(\mathrm{d}_{3}\right)$ & {$[\mathrm{mm}]$} & 0.023 & 0.025 \\
Length $\left(L_{1}\right)$ & {$[\mathrm{mm}]$} & 3.89 & 0.31 \\
Width $\left(d_{1}\right)$ & {$[\mathrm{mm}]$} & 0.022 & 0.024 \\
Fibre Coarseness $(c s)$ & {$[\mathrm{mg} / 100 \mathrm{~m}]$} & 17 & 17 \\
Crowding number $0.3 \%\left(N_{C W}\right)$ & {$[-]$} & 134 & $<1$ \\
Crowding number $0.1 \%\left(N_{C W}\right)$ & {$[-]$} & 45 & $<1$ \\
\hline
\end{tabular}


According to Kerekes (Kerekes, 2006) and the report by Celzard et al. (Celzard et al., 2009), we expect flocculation for the $\mathrm{CF}$ with a nominal length of $4 \mathrm{~mm}$, however, free fibre movement for $\mathrm{CF}$ with a nominal length of $0.3 \mathrm{~mm}$.

The density of the soaked CF is not straight forward to determine. We calculated the density from the measured water retention value according to ISO 23714:2014: fibres were suspended in water and water was removed by centrifuging at $3000 \mathrm{~g}$. The residual water was determined by thermo-gravimetric measurements. We found a water retention value of $80 \%$. With the density of pure cellulose of $1500 \mathrm{~kg} / \mathrm{m}^{3}$ and the density of water of $1000 \mathrm{~kg} / \mathrm{m}^{3}$, the density ratio of the soaked CF to the fluid density is ca. 1.3, a value also reported by Kvick (Kvick, 2014).

\subsection{Fibre Residence Time Distribution}

Laitinen et.al. (Laitinen et al., 2011) states the maximum mass concentration of fibre suspension for separation in the TFF as $0.5 \%$. Above this concentration, fibres form a coherent network hindering the separation process. The settings of Laitinen et al. were a fibre suspension concentration of $0.3 \%$, a pipe length of $100 \mathrm{~m}$ with an inner diameter of $0.016 \mathrm{~mm}$. The flow rate was set to $5.7 \mathrm{~L} / \mathrm{min}$. Coil diameter and design details of the utilities, e.g., the feed section, were not reported in this previous work.

Our validation experiments with the TFF were performed with SP at concentrations of $0.5 \%$ and $0.25 \%$, as well as at flow rates of $2.5 \mathrm{~L} / \mathrm{min}$ and $5.2 \mathrm{~L} / \mathrm{min}$. RT measurements with $\mathrm{CF}$ were performed at a concentration of $0.3 \%$. Table 2 lists the experimental settings.

Table 2: Settings for fibre residence time measurement. Flow rate, concentration, and material are changed in the experiment.

\begin{tabular}{ccccc}
\hline Experiment ID & $\begin{array}{c}\text { Flow rate } \\
{[\mathrm{L} / \mathrm{min}]}\end{array}$ & $\mathrm{Re}$ & Material & $\begin{array}{c}\text { Concentration } \\
{[\%]}\end{array}$ \\
\hline SP-0.25-3316 & 2.5 & 3316 & $\mathrm{SP}$ & 0.25 \\
SP-0.50-3316 & 2.5 & 3316 & $\mathrm{SP}$ & 0.50 \\
CF-0.3-3316 & 2.5 & 3316 & $\mathrm{CF}$ & 0.30 \\
SP-0.25-6926 & 5.2 & 6926 & $\mathrm{SP}$ & 0.25 \\
\hline
\end{tabular}


Manuscript: Mechanistic Understanding of Size-Based Fibre Separation in Coiled Tubes

\begin{tabular}{lllll} 
SP-0.50-6926 & 5.2 & 6926 & SP & 0.50 \\
CF-0.3-6926 & 5.2 & 6926 & CF & 0.30 \\
\hline
\end{tabular}

Experimental results were compared to the empirical RT correlation provided by Laitinen et.al. (Laitinen et al., 2011). They fitted the parameters of the correlation to different sized and shaped particles and fibres. The longest fibre length was $3.4 \mathrm{~mm}$. We neglected higher order elements for the fibre width and fibre thickness of the original correlation. The reference time was the mean residence time calculated from the fluid bulk velocity $u_{b u l k}$ and the tube length. The reference length was the tube diameter $d$. Equation (5) presents the adapted correlation. Parameter A is an offset, which is independent of fibre length, and was fitted to our results. All other parameters defining the shape of RT were taken from the original paper (see Table 3).

$$
\tau=A_{L}+B_{L} \cdot l+C_{L} \cdot c+D_{L} \cdot c+E_{L} \cdot l^{2}
$$

Table 3: Parameters adapted from the experimental model proposed by Laitinen (Laitinen et al., 2011). The parameters are presented for the non-dimensional equation (5).

\begin{tabular}{ccccc}
\hline $\mathrm{A}_{\mathrm{L}}$ & $\mathrm{B}_{\mathrm{L}}$ & $\mathrm{C}_{\mathrm{L}}$ & $\mathrm{D}_{\mathrm{L}}$ & $\mathrm{E}_{\mathrm{L}}$ \\
\hline 0.8150 & -0.8582 & -0.1114 & -3.9090 & 1.8023 \\
\hline
\end{tabular}

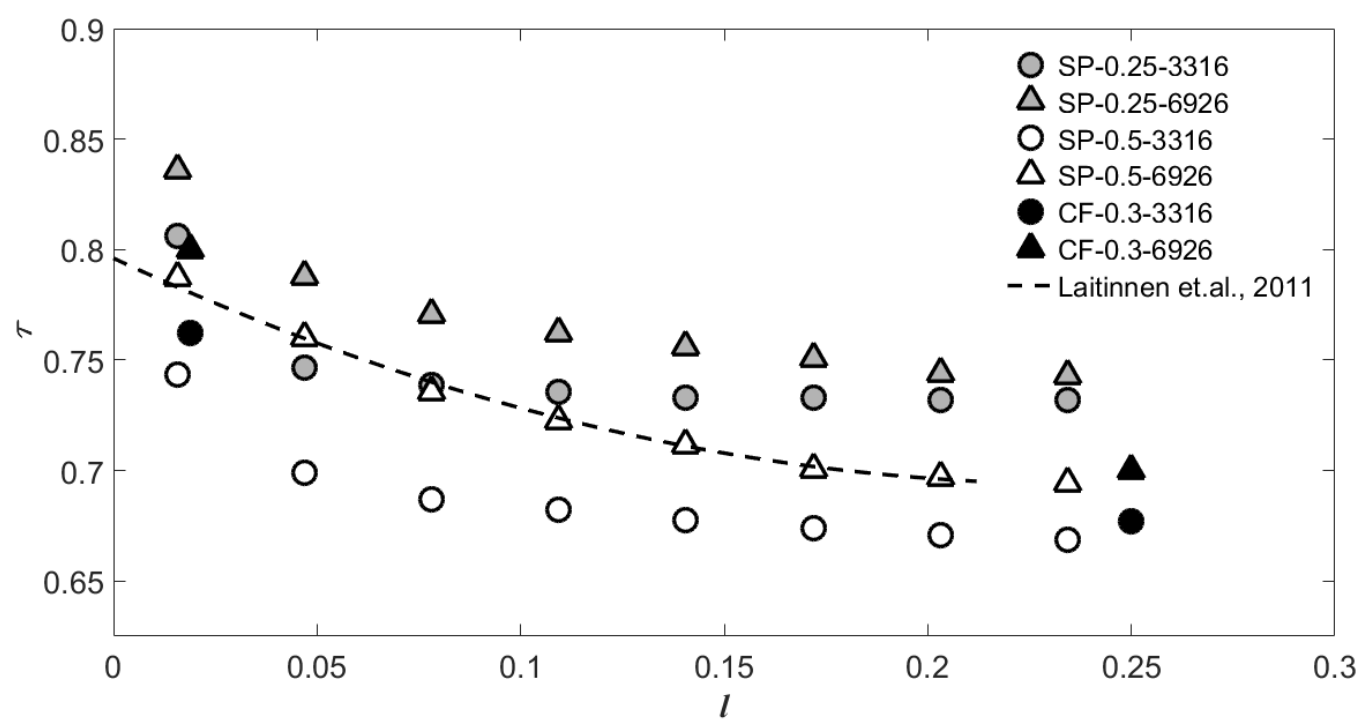

Figure 3: Fibre residence time $\tau$ vs. fibre length $l$ for sulfite pulp and synthetic cellulose fibres, as well as comparison to an empirical equation from literature (Laitinen et al., 2011). 
Figure 3 compares the experimentally measured non-dimensional RT $\tau$ in dependence of the dimensionless fibre length $l$ to each other and to literature data. In our experiment, fibres left the TFF at $\tau<0.9$. This means, that the collected fibres were faster than the average fluid velocity. Experimental results follow the same trend as the empirical equation. However, we found that $\tau$ per fibre length differed in dependence on $R e$ and the concentration. $\tau$ was lower for higher suspension concentrations (compare SP-0.25-3316 and SP SP-0.5-6926) and lower Re numbers (compare SP-0.25-3316 and SP-0.25-6926).

Experiments with $\mathrm{CF}$ at a suspension concentration of $0.3 \%$ resulted in an RT similar to that for SP. We know from the calculation of the crowding number $N_{C W}$ (Table 1) that CFs $4 \mathrm{~mm}$ formed strong networks, whilst CFs $0.3 \mathrm{~mm}$ were suspended freely in the fluid. Hence, the longer fibres might appear as a single larger particle of low solid/fluid density ratio. Shorter fibres were well mixed by the secondary motion, resulting in an (on average) longer residence time. This argument is supported by our results for SP. For higher consistency, the fibre suspension was more prone to form flocs. Thus, the fibres were conveyed through the TFF as a large fibre floc, resulting in a shorter residence time compared to cases with a lower concentration of fibres.

\subsection{Effect of Fibre-Fibre Interaction on the Residence Time Distribution}

To evaluate the influence of fibre-fibre interaction on the RT, we blended suspensions of monodisperse CF $4 \mathrm{~mm}$ and CF $0.3 \mathrm{~mm}$ at different ratios. The total fibre concentration was held constant at $0.3 \%$. The resulting RT per fibre length from the mixture was compared to the RT of the corresponding mono-disperse fibre suspension (mono). Results are listed in Table 4, and are shown in Figure 4. Experiments have been performed at a $R e$ of 3316 (i.e., a flow rate of $2.5 \mathrm{~L} / \mathrm{min})$.

Table 4: Residence time for mono-disperse fibre suspension and two different mixtures of $\mathbf{4} \mathbf{~ m m}$ and $0.3 \mathrm{~mm}$ CF. $R e=3316$.

$\begin{array}{ccc}\begin{array}{c}\text { Experiment } \\ \text { ID }\end{array} & \begin{array}{c}\text { Relative amount } \\ \text { of } 4 \mathrm{~mm} \text { fibres }\end{array} & \text { Residence Time RT }\end{array}$

Volume Based Number Based $\quad 4 \mathrm{~mm} \mathrm{CF} \quad 0.3 \mathrm{~mm} \mathrm{CF}$ 


\begin{tabular}{ccccc}
\hline Mono & - & - & $0.677 \pm 0.019$ & $0.762 \pm 0.010$ \\
L25S75 & 0.25 & 0.02 & $0.698 \pm 0.012$ & $0.766 \pm 0.018$ \\
L75S25 & 0.75 & 0.17 & $0.693 \pm 0.014$ & $0.752 \pm 0.030$ \\
\hline
\end{tabular}

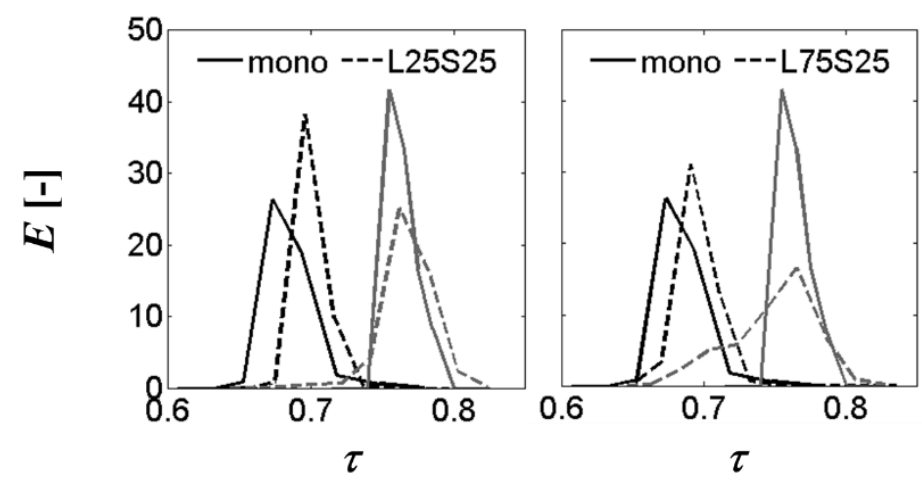

Figure 4: Normalized residence time distribution $E$ for four different fibre suspensions (two monodisperse, two bi-disperse; left panel: mass fraction of long fibres being 0.25 in the polydisperse mixture; right panel: 0.75). Lines for $4 \mathrm{~mm}$ fibres are colored black, for $0.3 \mathrm{~mm}$ fibres grey.

We found, that a small amount of long fibres, typical for fibre suspensions used in the pulp and paper industry, did not affect the RT of the small fibres. However increasing the amount of long fibres to $17 \%$ (based on the fibre number) was sufficient to drag short fibres, and thus change the mean RT, as well as the RT distribution. Following previous arguments we conclude, that short fibres were partially trapped in the network of long fibres.

\subsection{Mass Balance}

As we will demonstrate in Section 4 (Numerical Simulations) single fibres can have a residence time $\tau>1$ for $R e=3316$. We performed mass balance experiments where we collected all fibres up to a RT of $\tau \leq 1$, and $\tau \leq 1.5$ for $R e=3316$ and $R e=6926$. Thus, the overall sampling time was larger than the sampling time for RT measurements reported in the previous section. The fibre concentration was fixed at $0.10 \%$. This concentration is a compromise between a reduced concentration for having more dilute fibre flow and the necessity to collect a weighable amount of fibres. Between the runs, the TFF was flushed at the maximum flow rate of $12 \mathrm{~L} / \mathrm{min}(+/-$ $0.1 \mathrm{~L} / \mathrm{min}$ ) to ensure no carryover of fibres between experiments. The exit fraction, i.e., the mass of fibres collected compared to the mass of fibres fed, is summarized in Table 5. 
Manuscript: Mechanistic Understanding of Size-Based Fibre Separation in Coiled Tubes

Table 5: Exit fraction, amount of fibres collected compared to amount of fibres fed for different settings.

\begin{tabular}{cccc}
\hline $\mathrm{CF}$ & $\mathrm{Re}$ & $\mathrm{RT}$ & Exit Fraction \\
\hline $4 \mathrm{~mm}$ & 3316 & $\leq 1$ & 0.850 \\
$4 \mathrm{~mm}$ & 6926 & $\leq 1$ & 0.852 \\
\hline $0.3 \mathrm{~mm}$ & 3316 & $\leq 1$ & 0.631 \\
$0.3 \mathrm{~mm}$ & 3316 & $\leq 1.5$ & 0.667 \\
$0.3 \mathrm{~mm}$ & 6926 & $\leq 1$ & 0.834 \\
\hline
\end{tabular}

For none of the settings, total recovery was achieved. In accordance to the measured findings it was also visually recognized, that long fibres had been found leaving the TFF after the end of the experiment $(\tau>>1)$. In the range of parameters investigated, the behavior of $4 \mathrm{~mm}$ fibres was unaffected by changes of $R e$. The exit fraction of CF $0.3 \mathrm{~mm}$ was smaller than the exit fraction of CF $4 \mathrm{~mm}$ at Re 3316. Increasing Re increased the mass of recovered fibres, and sizeindependent results (for the exit fraction) were achieved. Increasing the sampling interval from $\tau \leq 1$ to $\tau \leq 1.5$ had no substantial effect on the amount of collected fibres at low $R e$. Thus, we observe that fibres are trapped for a long time in the coiled tube. Obviously, the reason why we encounter no total recovery of fibres within the operation time of the TFF must be a sedimentation effect. This will become clear after considering our simulation results, which indicate a strong effect of sedimentation on fibre trajectories for low $R e$. 


\section{Numerical Simulations}

Our experimental study, in accordance with literature, revealed a complex behavior of elongated particles in coiled suspension flow: due to the coiled geometry, secondary fluid motion acts on suspended particles, causing mixing, and hence affects the RT and the RT distribution. As a function of the concentration of elongated particles, their appearance in the flow can change from individual fibres to coherent networks, i.e., fibre flocs. For the following simulation study we limited the number of cases to dilute suspensions of elongated particles, and hence neglect fibre-fibre interaction. Thus, the case presented below reflect situations for which $N_{C W}<1$. In order to understand the effect of fluid flow on the fibre trajectories, we varied the Reynolds number $R e$ and curvature $\kappa$.

The polydisperse fibre population was modeled as a set of rigid cylinders with spherical endcaps (Figure 5, left), i.e., so-called "spherocylinders" (Lu et al., 2015). Fibre geometry, i.e., the length and aspect ratio of the spherocylinders, was chosen to model typical fibres in pulp and paper applications. The spherocylinder is defined by its length $l$ and its diameter $c$. The aspect ratio $\lambda$ is defined as: $\lambda=l / c . \delta_{\text {rough }}$ presents the length of the fibre roughness, i.e. fibre fibrils and adds a damping region in the simulation. The fibre is associated with a local coordinate system x", y", z".
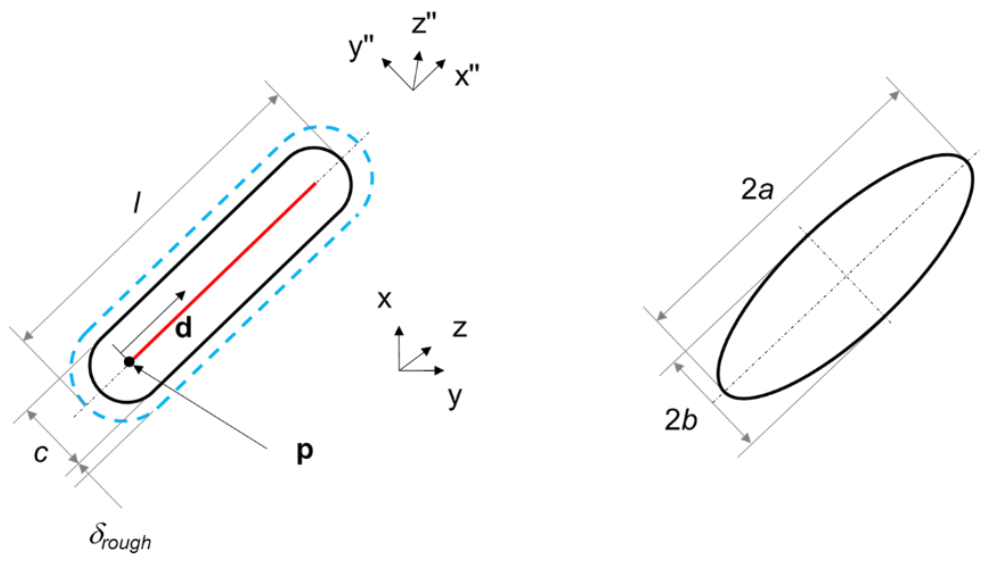

Figure 5: Left panel: sphero-cylinder shaped stiff fibres with half-sphere endcaps having length $l$ and diameter $c$. The fibre is associated with a local coordinate system $x^{\prime \prime}, y^{\prime \prime}, z^{\prime \prime}$ with its origin at $p . z$ is the orientation of the fibre. Right panel: prolate spheroid with dimensions $a$ and $b$ as the major and minor semi-axis, respectively.

Fibres, represented as spheroid (Figure 5), were modeled to interact with the fluid via hydrodynamic forces, and a Stokes flow regime is assumed (Kim and Karrila, 2005). The fibre 
model coincides with the presentation in Lindström and Uesaka (Lindström and Uesaka, 2007), with the exception that fibres are assumed to be rigid. Hydrodynamic properties of the spherocylinder and the spheroids are similar when (i) the major semi-axis $a$ of the spheroid is identical with the half-length of the spheroid $(a=l / 2)$, and (ii) the minor semi-axis $b$ is: $b=\frac{1}{2.48} c \sqrt{\ln (l / c)}($ Cox, 1971). A detailed description of our modelling approach including the validation of the fibre-fibre, fibre-wall, and fibre-fluid interaction is given by RedlingerPohn, et.al. (Redlinger-Pohn et al., 2016).

Fibres are introduced into the fully developed flow field, and their effect on the flow field is neglected. This is a common approach, and valid for dilute suspensions of single fibre flow (Marchioli and Soldati, 2013; Marchioli et al., 2010; Mortensen et al., 2008a, 2008b). Note, that neglecting the effect of the fibres on fluid flow might be problematic for more concentrated suspension, as identified in previous work (Krochak et al., 2009). Fluid motion in the toroidal domain was calculated using the open source code OpenFOAM, version 2.3.0 (The OpenFOAM Foundation, 2014). An appropriately fine mesh resolution, and second-order accurate numerical schemes were used in our simulations. The mesh study was guided by work of Di Liberto et.al. (Di Liberto et al., 2013) and Di Piazza and Ciofalo (Di Piazza and Ciofalo, 2011). Single-phase toroidal fluid flow, the results from the mesh dependency study, the influence of the torus domain size, as well as comparison to literature data from Hüttl and Friedrich (Hüttl and Friedrich, 2000) are given in the electronical Annex (see Electronic Annex in the online version of this article).

\subsection{Simulation Settings}

The dimensionless continuity and Navier-Stokes equation were solved in an orthogonal Cartesian coordinate system $(\mathrm{x}, \mathrm{y}, \mathrm{z})$ :

$$
\begin{gathered}
\frac{\partial u_{i}^{*}}{\partial x_{i}{ }^{*}}=0, \\
\frac{\partial u_{i}^{*}}{\partial t^{*}}+u_{j}^{*} \frac{\partial u_{i}^{*}}{\partial x_{i}^{*}}=-\frac{\partial p_{s}^{*}}{\partial x_{i}^{*}}+\frac{1}{\operatorname{Re}} \frac{\partial^{2} u_{i}^{*}}{\partial x_{i}^{*} \partial x_{j}{ }^{*}}+g_{i}{ }^{*} .
\end{gathered}
$$


The pipe diameter $d$ and the mean bulk velocity $u_{b u l k}$ of the fluid are set to unity in all our simulations. Thus, we focus on a non-dimensional system with the reference length being $d$, and the reference speed being $u_{b u l k}$. The reference pressure was chosen as $\rho U^{2}$, the viscosity was adjusted to yield the desired Reynolds number of the flow. The pressure drop $p_{s}$ in the flow direction was balanced with an additional source term.

Figure 6 presents the half-torus in toroidal and Cartesian coordinates. $D$ is the coil diameter. $r$ and $\varphi$ describe the position of a point in the cross section of the torus, $R$ and $\Theta$ the radial position and azimuthal angle the position of the point in the torus, respectively. I and O denote the inner and outer bend of the pipe with respect to the torus. UH and LH denote the upper half of the torus cross section, and the lower half of the torus cross section.

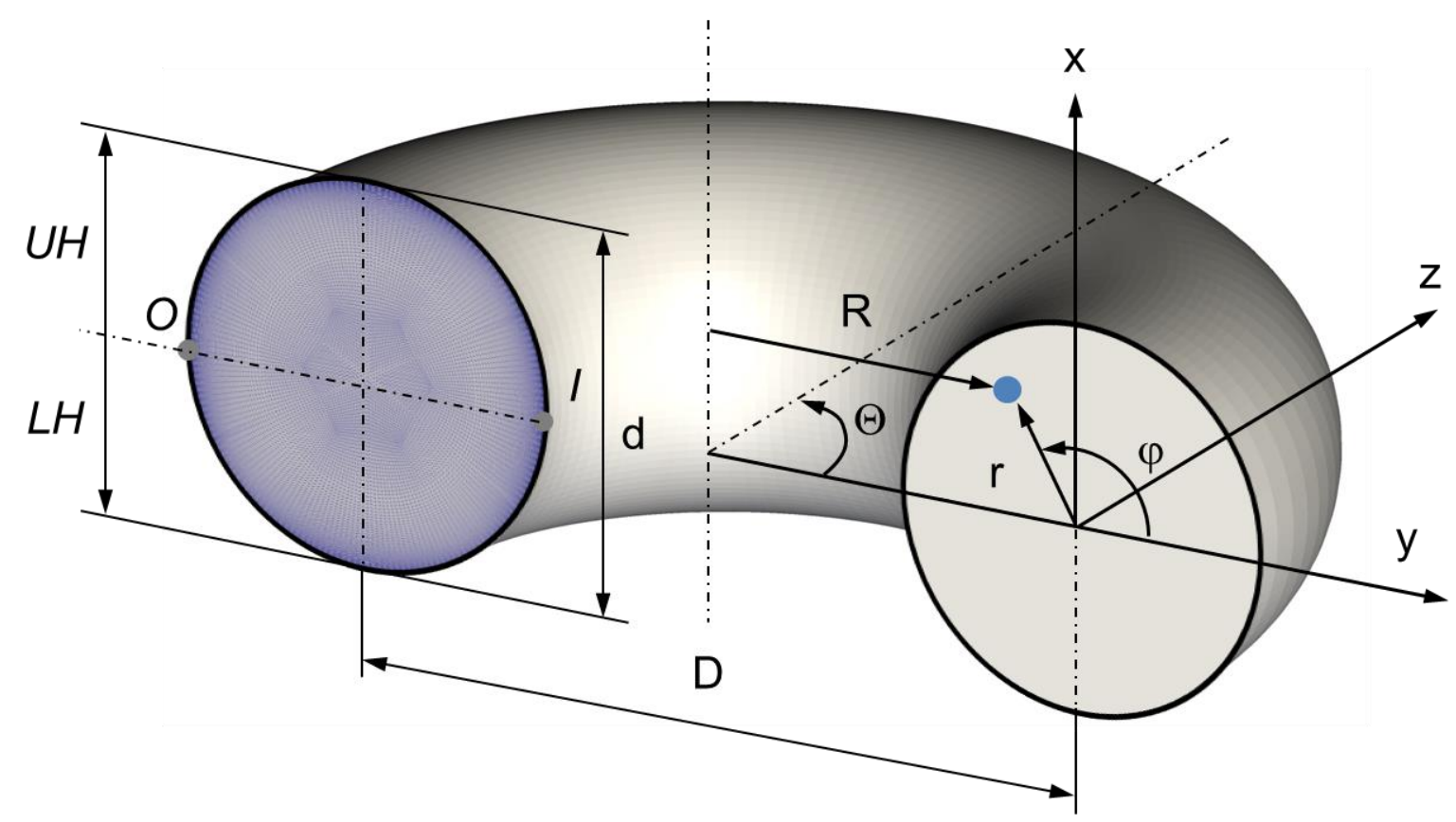

Figure 6: Schematic representation of the half torus in the Cartesian $(x, y, z)$ and toroidal $(r, \varphi, \Theta)$ coordinate system. $D$ and $d$ present the torus and pipe diameter. $I$ and $O$ denote the inner and outer wall of the torus. Left cross section of the half torus presents the cross sectional mesh. LH and UH denotes the lower half and the upper half of the torus cross section.

Two-phase simulations are performed for two different curvatures $\kappa$, and two different Reynolds numbers $R e$ resulting in a set of three Dean numbers $D a$. Settings and maximum value of the secondary motion for the fluid motion are given in Table 6. $u_{x}$ presents the part of the secondary motion parallel to the direction of gravity. 
Manuscript: Mechanistic Understanding of Size-Based Fibre Separation in Coiled Tubes

Table 6: Secondary motion $\boldsymbol{u}_{\text {sec }}$ and velocity parallel to gravitational force $\boldsymbol{u}_{x}$ for the simulated two-phase cases TFF-7, TFF-10, and TFF-1 differing in the curvature $\kappa$ and the Reynolds number $R e$.

\begin{tabular}{cccccc}
\hline Case & $\kappa$ & $R e$ & $D a$ & $u_{\text {sec }}$ & $u_{x}$ \\
\hline TFF-7 & 0.1 & 3316 & 1048 & 0.2133 & 0.1334 \\
TFF-10 & 0.043 & 3316 & 688 & 0.1415 & 0.0874 \\
TFF-1 & 0.1 & 6926 & 2190 & 0.2113 & 0.1314 \\
\hline
\end{tabular}

Corresponding snapshot of the stream-wise motion and secondary motion in the tubes cross section are shown in Figure 7.

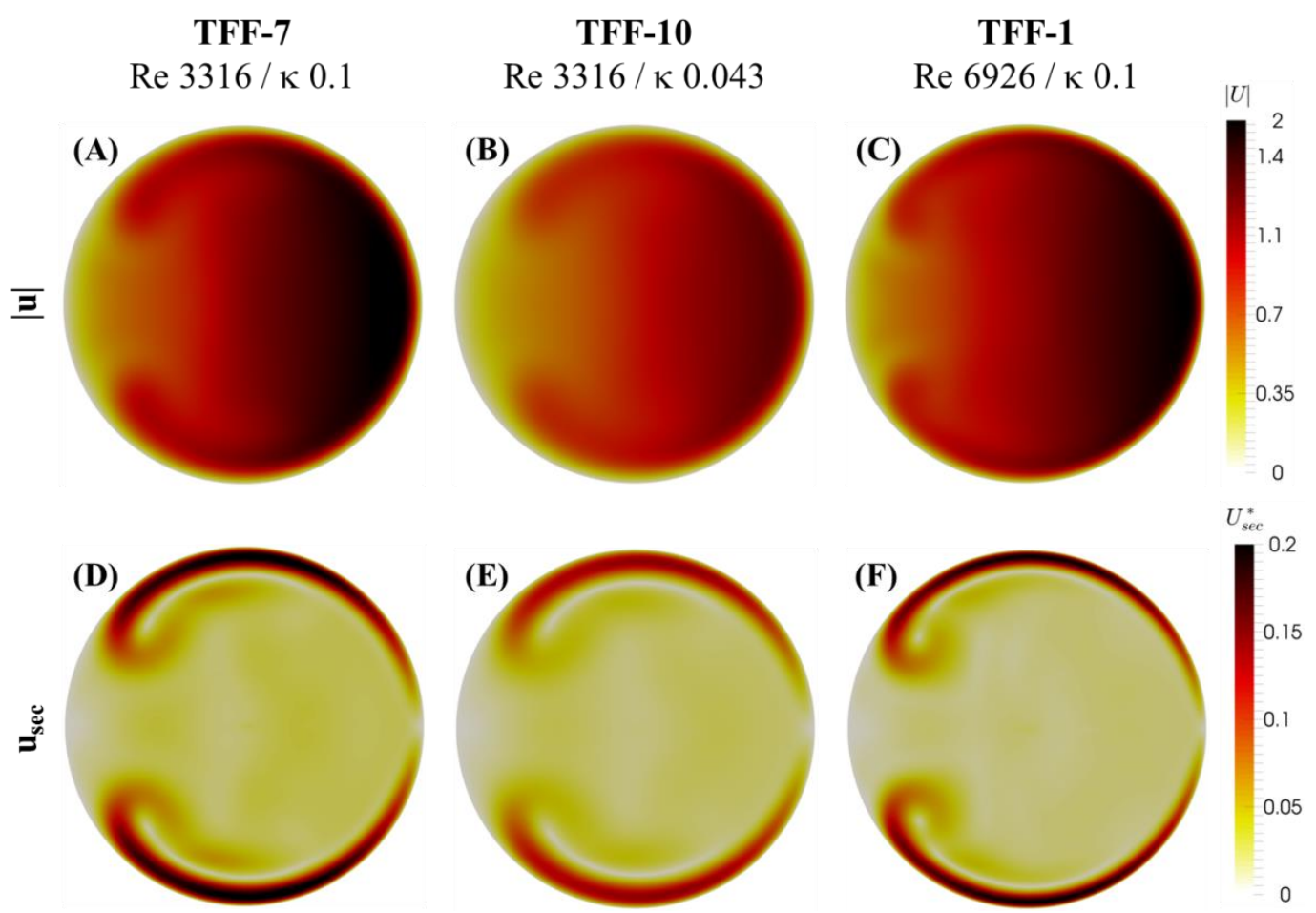

Figure 7: Upper panel: time averaged velocity magnitude (A to C), indicating the deflection of the flow towards the outer bent. Lower panel: secondary flow in the cross section of the pipe (D to F, data was time-averaged in case oscillations occurred). Panels present results for TFF-7 (left panel), TFF-10 (centre panel), and TFF-1 (right panel).

The intensity of the secondary flow was mainly affected by the curvature, and only to a much smaller extent by the Re. We observe that the width of the secondary flow streak (i.e., the width of the secondary motion in the cross section along the pipe wall) increased slightly with decreasing $R e$. Established fluid flow field is in accordance with literature as shown in the electronical Annex (see Electronic Annex in the online version of this article). 
The length of the longest simulated fibre was set to $0.25 \cdot d$. This is in accordance with the experiments presented in Section 3 (see Table 1). Three types of fibres (Table 7) were considered in the simulations.

Table 7: Geometrical properties of the simulated fibres in relation to the experiment.

\begin{tabular}{lccc}
\hline Aspect ratio $\lambda[-]$ & 160 & 40 & 2 \\
Simulation, $l[-]$ & 0.25 & 0.0625 & 0.003125 \\
Experiment, $L[\mathrm{~mm}]$ & 4 & 1 & 0.05 \\
\hline
\end{tabular}

The particle-to-fluid density ratio was set to 1.3 for cellulose fibres. In one additional simulation experiment (TFF-7GF) we modelled glass fibres having a density ratio of 2.6, i.e., two times the density ratio of cellulose fibres. Fibres were positioned uniformly distributed in the cross section of the torus. Fibres of different fibre type (i.e., aspect ratio) shared the same starting position to avoid any bias originating from initial conditions. Furthermore, orientation of fibres was random. Simulations were performed with 3096 fibres per fibre type, i.e., 9288 particles were tracked in total.

\subsubsection{Scaling Analysis}

Previous literature indicates that three effects might be responsible for fibre positioning in the cross section of the torus: (i) fibre-wall interactions of flipping fibres occurring close to the torus' outer wall, (ii) sedimentation effects, and (iii) secondary motion that introduces cross sectional mixing. To estimate the required simulation time, the duration for completing one Jeffery Orbit $\tau_{J O}$ (at the position of maximum shear in the fluid), and the time to sediment from the pipe top to the bottom was calculated for different settings (Table 8). The time to complete one Jeffery Orbit was calculated using equation (3). The dimensionless fluid shear rate is 33.67. The terminal settling velocity $v_{s}$, as well as the resulting sedimentation time, $t_{s}$ was calculated for fibre orientation normal and parallel to the direction of gravity. Calculation of the drag force and thus the settling velocity of a sedimenting fibre is given in Redlinger-Pohn et.al. (RedlingerPohn et al., 2016). With the reference velocity $\left(u_{b u l k}\right)$ and reference length $(d)$ chosen, the gravity $g$ was adjusted in the simulation to match the experimental Froude number $\operatorname{Fr}\left(\operatorname{Fr}=u_{\text {bulk }} / \sqrt{g d}\right.$ ). The Froude number resulted to $F r=0.523$, and $F r=1.093$ for cases $\operatorname{Re}=3316$, and $\operatorname{Re}=$ 6926, respectively. Obviously for higher inertial effects, which is expressed by larger $R e$, the 
Manuscript: Mechanistic Understanding of Size-Based Fibre Separation in Coiled Tubes

influence of gravitation is reduced which is expressed by larger values for Fr. Sedimentation effect is thus lower. The reference time is the convective time scale $d / u_{b u l k}$.

Table 8: Terminal settling velocity and resulting sedimentation time for fibres in the torus' cross section.

\begin{tabular}{|c|c|c|c|c|c|c|c|c|}
\hline$R e$ & $F r$ & $\lambda_{\text {spheroid }}$ & $\tau_{J O}$ & $\lambda_{\text {cylinder }}$ & $t_{\text {sed,normal }}$ & $t_{\text {sed.parallel }}$ & $\begin{array}{c}v_{s, \text { normal }} \\
\cdot 10^{-3}\end{array}$ & $\begin{array}{c}v_{s, p a r a l l e l} \\
\cdot 10^{-3}\end{array}$ \\
\hline \multirow[t]{3}{*}{3316} & 0.523 & 88 & 16.4 & 160 & 144 & 88 & 6.92 & 11.41 \\
\hline & & 26 & 4.8 & 40 & 254 & 163 & 3.94 & 6.12 \\
\hline & & 3 & 0.6 & 2 & 2598 & 2115 & 0.38 & 0.47 \\
\hline \multirow[t]{3}{*}{6926} & 1.088 & 88 & 16.4 & 160 & 299 & 182 & 3.34 & 5.51 \\
\hline & & 26 & 4.8 & 40 & 525 & 338 & 1.90 & 2.96 \\
\hline & & 3 & 0.6 & 2 & 5382 & 4380 & 0.19 & 0.23 \\
\hline
\end{tabular}

The above scaling analysis results indicate that fibres located in the region of high shear rates (i.e., close to the outer torus' wall) would theoretically flip once per half-length of the torus. Fibre-wall interaction hence plays a role, and will critically affect the fibres' movement in the torus. Also, the sedimentation velocity is in the range of several percent of the maximum upwards velocity $u_{x}$ (compare Table 8 with Table 6 ), and thus cannot be neglected.

\subsection{Results}

Simulations ran for 60.4 dimensionless time units (dtu) for case TFF-1 (transient flow field), 418.4 dtu for case TFF-7 (steady-state flow field), 127.0 dtu for case TFF-7GF (increased density ratio of 2.6 to model glass fibres), and 445.7 dtu for case TFF-10 (steady-state flow field). Figure 8 presents the fraction of fibres for each fibre type in the upper half of the pipe cross section (UH, i.e., located between $\varphi=0$ and $\pi$ ) tracked over the simulated time. For case TFF-1 and TFF-7GF, fibres reached their steady-state value in a short time, i.e., within ca. 100 dtu. For TFF-7, and TFF-10 no steady-state value was obtained for fibre type $\lambda 40$. However, the tendency is obvious, whilst fibre type $\lambda=160$, and $\lambda=2$ reached stable fraction of fibres in the UH, fibre type $\lambda=40$ tends to be depleted. 


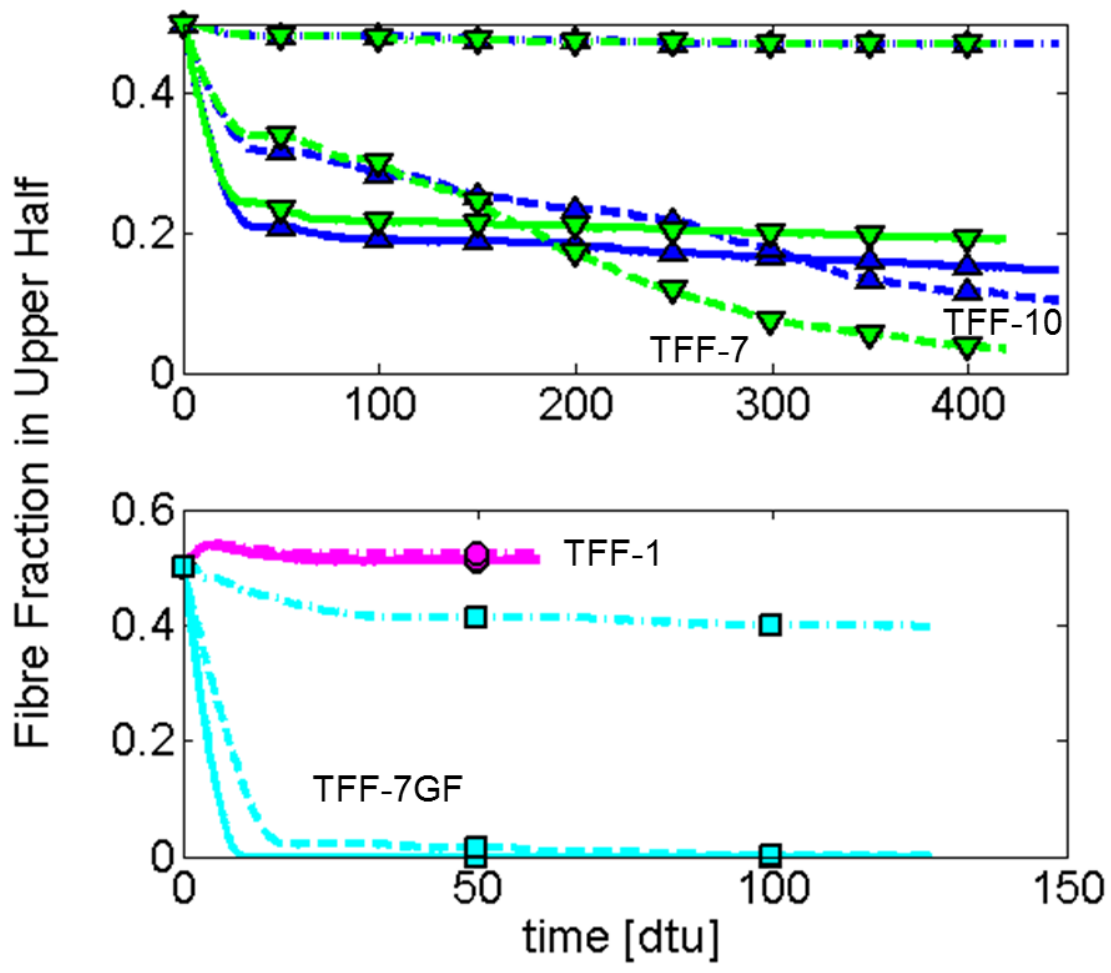

Figure 8: Fraction of fibres for each fibre type residing in the upper half of the cross section over the simulated time. Fibre types are labeled by line type: AR 160 (-----), AR 40 (- - - ), AR 2 (-.-.-.). Cases are labeled by color and symbols: TFF-7 (downward-pointing triangle, green) , TFF-10 (upward-pointing triangle, blue), TFF-1 (circle, magenta), and TFF-7GF (squares, cyan). The fibre position data was recorded every $0.1 \mathrm{dtu}$.

In the following chapters we discuss the fibre behavior and the fibre movement in the cross section, as well as evaluate the fibre orientation. We will then present result of the residence time distribution of the fibres based on our simulation results.

\subsubsection{Movement and Position of Fibres in the Cross Section}

Results were analyzed, and are discussed for every fibre type using Figure 9 to Figure 12. Fibre movement in the lower half of the tours cross section (LH, i.e., located between $\varphi=\pi$ and $2 \pi$ ) was found to be in accordance with literature (Palazoglu and Sandeep, 2004; Tiwari et al., 2006) that focused on spherical particles in coiled tube flow. We observe that fibres are driven towards the outer wall by the secondary motion (see Figure 7 lower panel), as well as centrifugal forces. At the outer wall, secondary flow and sedimentation caused fibres to settle towards the bottom. Then, the fibres are entrained into the secondary motion directed towards the inner wall. For fibres with $\lambda=160$ and $\lambda=40$, fibres accumulate at the wall between $\varphi=\pi$ and $3 / 4 \pi$ (see Figure 
9 to Figure 12, panel denoted as "Fibre Position"). In these regions our CFD simulations predict comparably low axial fluid velocities (compare Fehler! Verweisquelle konnte nicht gefunden werden. and Figure 7, upper panel), resulting in a slow fibre axial propagation through the TFF. Depending on the fibre size, fibres move at different orbits in the LH. Fibres with $\lambda=2$ are small enough to be nearly unaffected by the gravitational force, and hence are well dispersed in the toroidal flow.

Greater focus is now given to fibre movement in the UH. Specifically, we tracked 4 randomly chosen fibres, and present their preferred position and orbiting behavior in the cross section. To illustrate the integral behavior of the fibre cloud, the fibres' orbit position is presented as a contour plot of the fibre concentration.

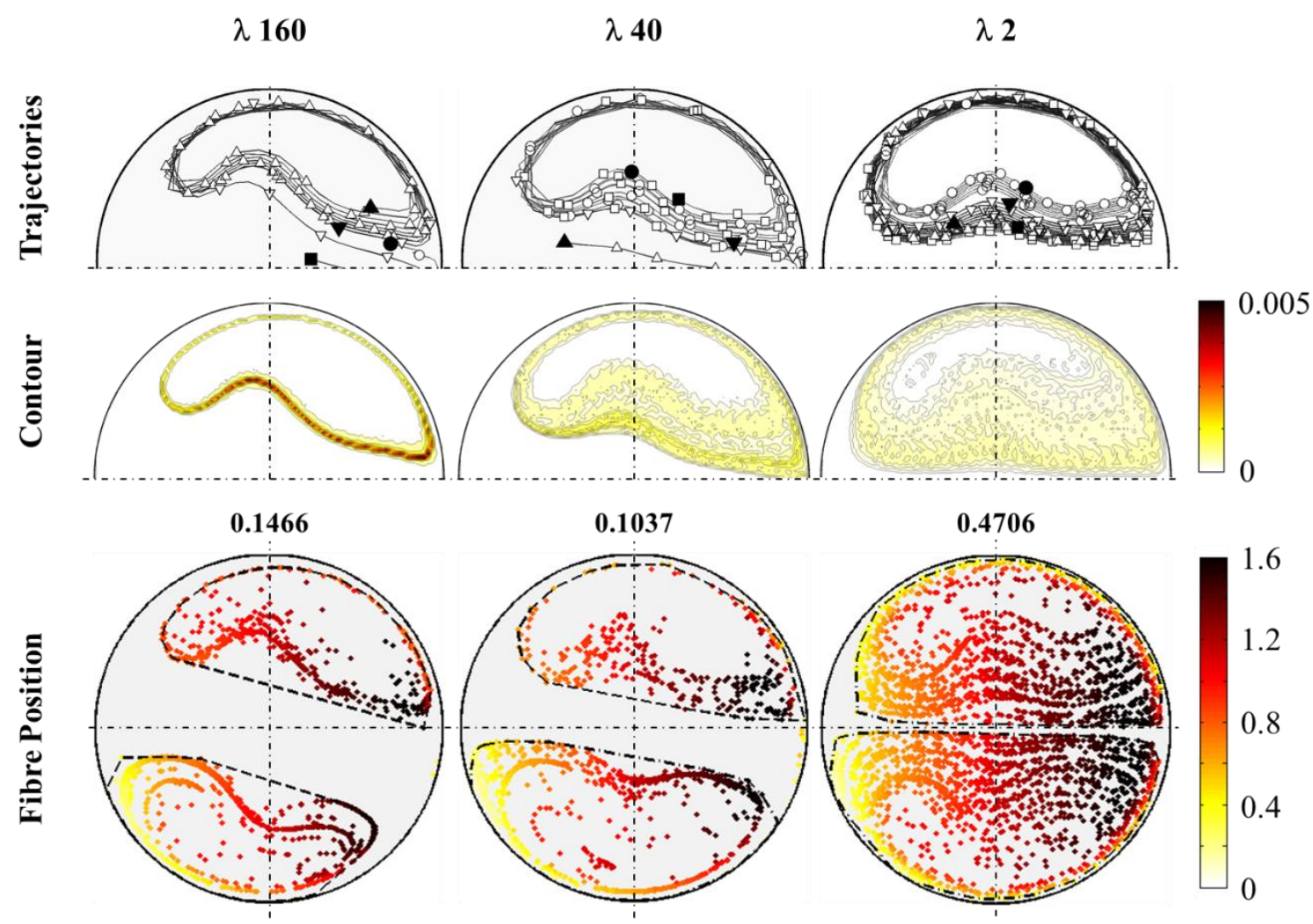

Figure 9: Results of simulation experiment TFF-10 $(R e=3316, \kappa=0.043)$ : fibre trajectories, contour plot of fibre position, and fibre position after $445.7 \mathrm{dtu}$. The contour plot presents the fibres' probability to be located at a certain position in the torus. Fibres in the bottom panel are colored by their axial velocity.

For TFF-10 $(R e=3316, \kappa=0.043$, see Figure 9$)$ and TFF-7 $(R e=3316, \kappa=0.1$, see Figure 10) we found similar results. Fibres drifted towards the outer wall driven by the secondary motion of the fluid (compare Figure 7, lower panel). Also, centrifugal forces cause later fibre transport, since the fibres' density is larger than that of the fluid. At the outer wall in the UH, the secondary 
flow of the fluid is pointing into the opposite direction of gravity. Fibres entrained into this secondary motion (in the UH) are carried upwards, and move in a counter-clockwise direction. Fibres with $\lambda=160$ and $\lambda=40$ are moving close to the tube wall from the outer side towards the inner side of the tube. At $\varphi=\pi / 2$, secondary motion changes direction and acts in the same direction as gravity. Once again settled to the torus center, fibres are driven towards the outer wall completing one orbit. From an analysis of the fibre trajectories we found that not all $\lambda=$ 160 and $\lambda=40$ fibres touch the outer wall in the UH before settling to the LH. Whether these fibres touch the wall or not (in the UH) depends on the position of the fibre in the orbit, which is affected by their sedimentation behavior. In summary, the fibres' sedimentation speed, as well as wall interaction are key to explain their trajectories.

Most important, we find from an inspection of the trajectories and the contour plot that $\lambda=160$ fibres are significantly more concentrated in a thin band. The reason for this peculiar behaviour is the fact that $\lambda=160$ fibres are heavier, and hence sediment faster (see Table 8, $v_{s, \text { parallel, }, 160=}$ $\left.6.92 \cdot 10^{-3}, v_{s, \text { parallel }, \lambda 40}=3.94 \cdot 10^{-3}\right)$. Thus, when located at a similar horizontal position, $\lambda=160$ fibres will sediment to a lower vertical position compared to shorter (and hence lighter) fibres. Thus, larger fibres are able to follow the secondary motion in the Dean vortices more tightly: Once sedimented for a certain vertical distance, long fibres enter the upper Dean vortex, where the fluid's secondary motion changes direction from vertical down- to up-flow. Interestingly, the magnitude of the vertical up-flow is larger than the settling velocity of the $\lambda=160$ fibres, and consequently these long and heavy fibres are gradually lifted upwards again. Clearly, this interplay of sedimentation and entrainment in a secondary flow (i.e., the upper Dean vortex), as well as the infrequent flipping of long fibres near the wall are the reason for the concentrated orbiting behaviour of these fibres.

From inspections of movies illustrating the simulation results, as well as the local fibre concentration plot, it is clear that the horizontal speed of fibre migration from the outer wall towards the inner wall is significantly larger compared to the opposite direction. This results into a rather steep angle of the fibre trajectories for the $\lambda=160$ fibres (see Figure 9 and Figure 10). Another observation is that on the way from the outer wall to the inner wall fibres concentrate in a thin band. Thus, fibre-wall collisions caused by fibre flipping events are too infrequent to effectively disperse long fibres in the tube's cross section. 
We observe that $\lambda=40$ fibres settle slower. As a result, fewer of them enter the upper Dean vortex, and hence fewer fibres get a vertically up-lift by the Dean vortex. Hence, $\lambda=40$ fibres spread over a larger vertical distance (see contour plot and the fibre position in Figure 9 and Figure 10), and have a lower vertical position. This results into the surprising finding that $\lambda=$ 40 fibres have a higher chance to settle into the LH. Following the trend presented in Figure 8, we can assume that this will result in a total depletion of $\lambda=40$ fibres from the UH, whilst a stable amount of $\lambda=160$ fibres remain in the UH. In summary, these results are rather surprising, and are explained by the interplay of the fibres' settling velocity and the secondary fluid motion.

The lightest, i.e., the $\lambda=2$ fibres were nearly unaffected by gravitation. From the fibre position (Figure 9 and Figure 10) we found similar position of $\lambda=2$ fibres in the UH and LH. Only near the inner wall, we found fewer fibres in the UH. In summary, $94 \%$ of the fibres initially placed in the $\mathrm{UH}$ remained there. We can thus argue that $\lambda=2$ fibres were small enough to be almost perfectly mixed in the tube by the secondary motion of the fluid.

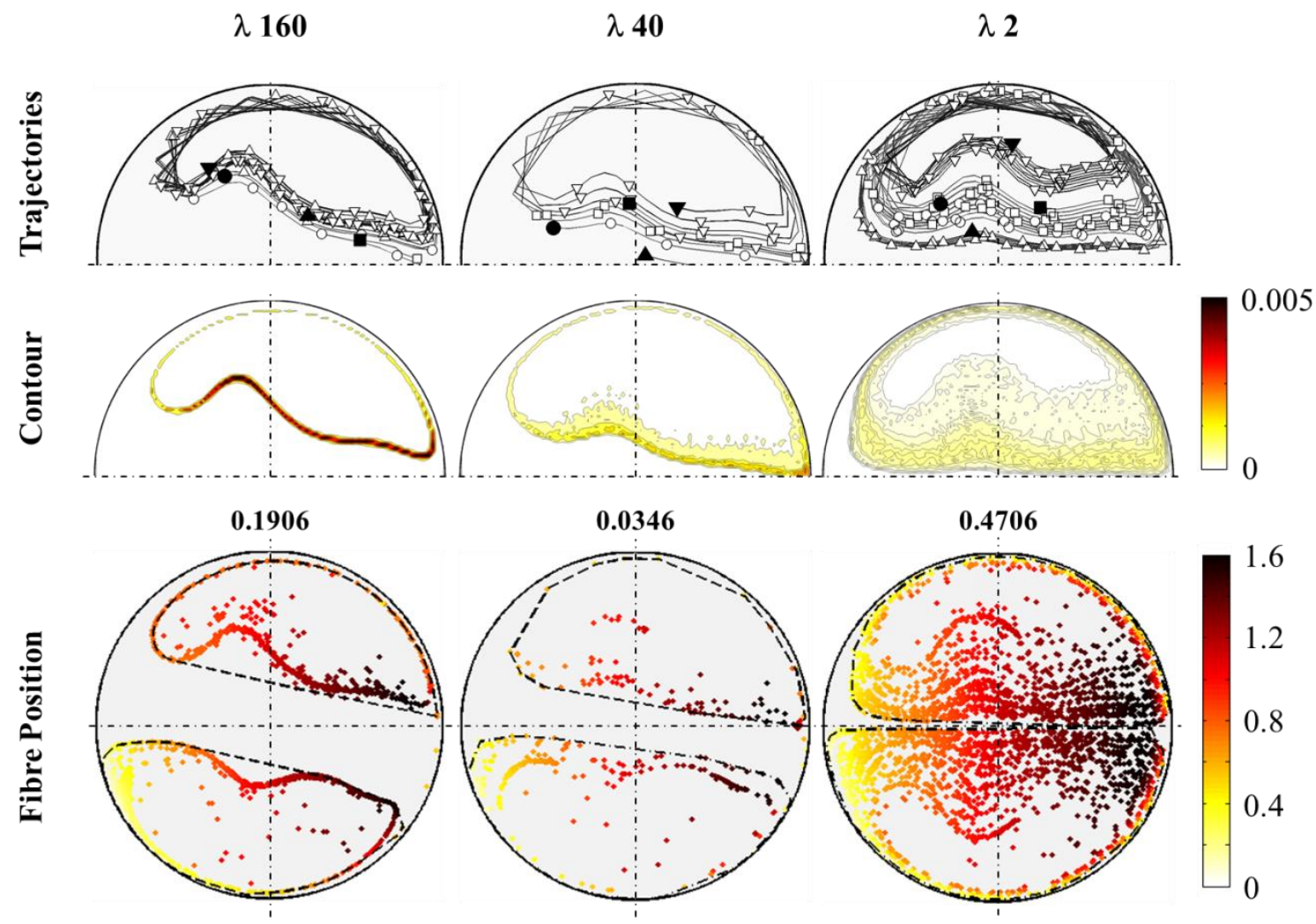

Figure 10: Results of simulation experiment TFF-7 $(R e=3316, \kappa=0.1)$ : fibre trajectories, contour plot of fibre position, and fibre position after 418.4 dtu. Labelling is identical to that used in Figure 9. 
When comparing TFF-10 (Figure 9) and TFF-7 (Figure 10) we also found slight differences in the results, which is attributed to differences in the curvature (i.e., $\kappa 0.043$ vs. $\kappa 0.1$ ). The Dean number was 688 and 1049 for TFF-10, and TFF-7, respectively. Thus, the extent of the secondary motion was larger for TFF-7. Thus, (i) $\lambda=160$ fibres experienced a greater upwards lift by the Dean vortex, and (ii) more $\lambda=40$ fibres were pushed towards the inner wall. Consequently, the fraction of $\lambda=160$ fibres in the UH was larger, and the fraction of $\lambda=40$ fibres in the UH was lower in TFF-7, compared to TFF-10.

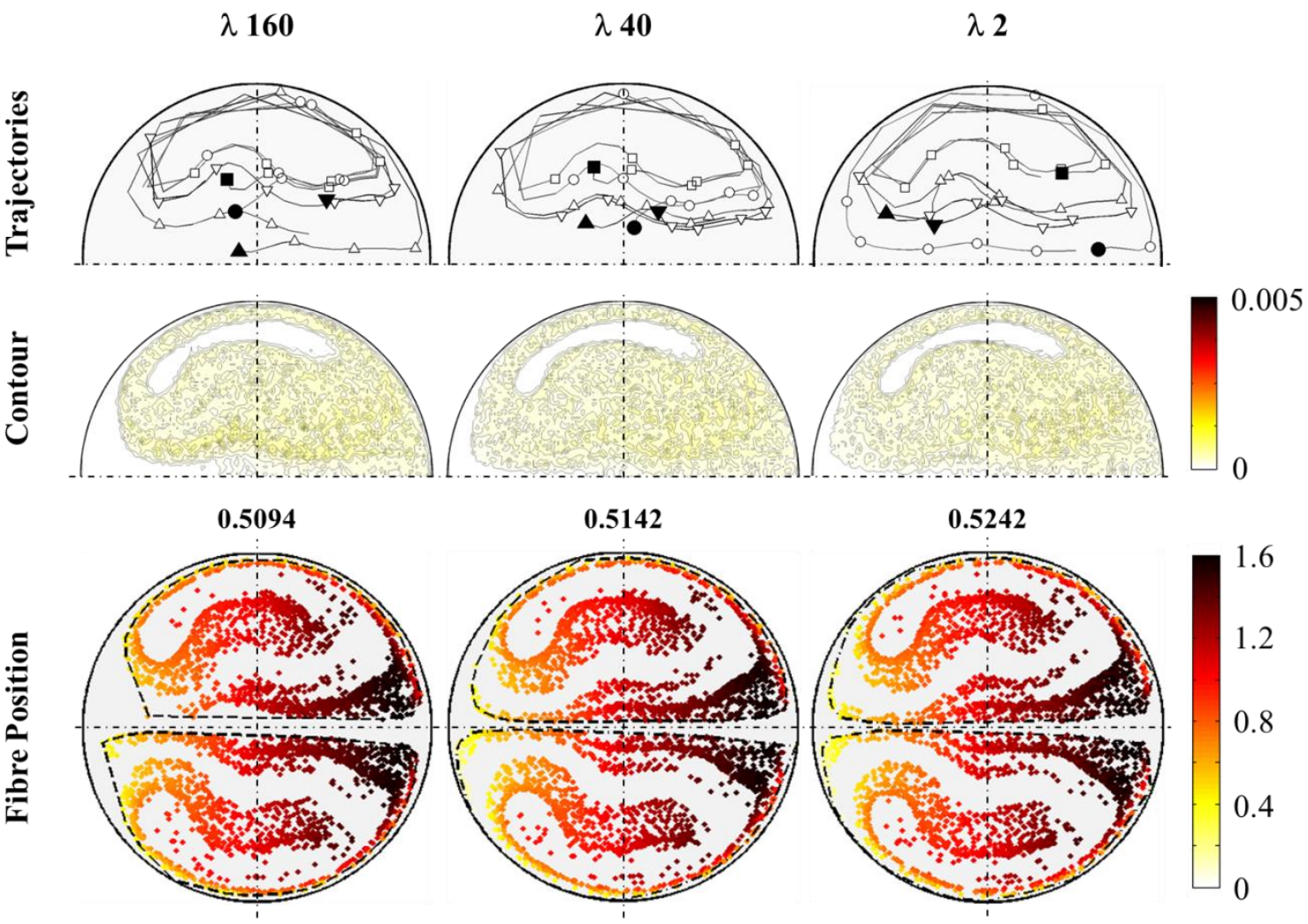

Figure 11: Results of simulation experiment TFF-1 $(R e=6926, \kappa=0.1)$ : fibre trajectories, contour plot of fibre position, and fibre position after 60.4 dtu. Labelling is identical to that used in Figure 9.

At higher Froude number what correlates to a higher Reynolds numbers, the ratio of the gravitational force to the centrifugal force is lower. Consequently, the ratio of the settling velocity to a typical speed of the secondary motion is lower. Specifically, we find for simulation experiment TFF-1 $(R e=6926, \kappa=0.1$, see Figure 11) that all fibres (independent of their size and mass) are well mixed in the cross section. Small differences were only found for the inner wall, with $\lambda=2$ fibres being transported closer to the inner wall compared to $\lambda=40$ and $\lambda 160$ fibres. Interestingly, we notice a small increase of the relative amount of fibres in the UH for very early times (i.e., for $10 \mathrm{dtu}$ as can be seen in Figure 8). We attribute this finding to the fact 
of the oscillatory nature of the Dean vortices at the specific Reynolds number studied. This means that the Dean vortices in the upper and lower half of the torus appear and vanish in a repeating oscillating manner, in agreement with literature (Di Piazza and Ciofalo, 2011). Fibres at the mid-plane might be affected and pushed either into the UH or the LH. Hence, over a longer period of time, we expect these fluctuations to even out. Interesting to note is that we found an area completely void of fibres (see Figure 11, centre panel). Similar findings can be seen for the simulation experiments TFF-10 and TFF-7, especially for $\lambda=2$, however, to a lower extent. The area coincides with the area of zero secondary motion (Figure 7, lower panel) separating the fluid's secondary motion directed towards the inner wall from the secondary motion directed towards the outer wall.

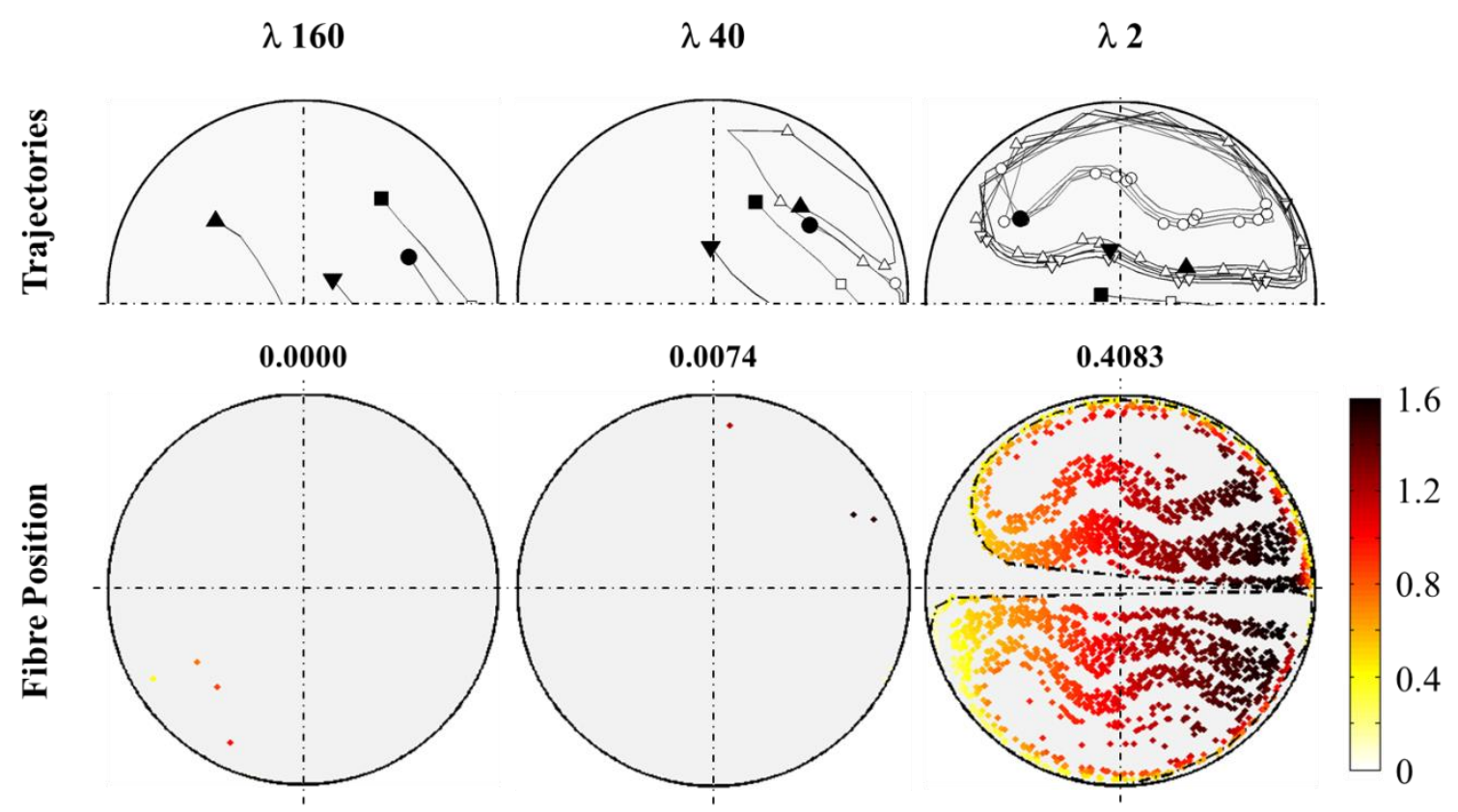

Figure 12: Results of simulation experiment TFF-7GF $(R e=3316, \kappa=0.1)$ : fibre trajectories, contour plot of fibre position, and fibre position after 127.0 dtu. Labelling is identical to that used in Figure 9.

To highlight the importance of the ratio between fibre settling velocities to the secondary motion we ran simulations of TFF-7 with a significantly higher fibre density (i.e., simulation experiment TFF-7GF, $R e=3316$, and $\kappa=0.1$, see Figure 12). From the trajectories we can see, that $\lambda=160$ and $\lambda=40$ fibres nearly instantaneously settle to the LH. Once arrived in the LH, fibres accumulate at the tube wall in a region characterized by $\varphi=\pi \ldots 3 / 4 \pi . \lambda=2$ fibres were more affected by gravity compared to the simulation experiment TTF-7. Approximately $20 \%$ of the initial fibres placed in the UH settled towards the LH. However, they were still fairly well dispersed over the cross section of the toroid. 


\subsubsection{Fibre Orientation}

Fibre orientation in the tube with respect to the direction of gravity defines the fibre resistance to sedimentation, and thus dictates the fibres' settling velocity (see the sedimentation velocity in Table 8). For the analysis of the fibre orientation, a wall-based reference coordinate system $x^{\prime}, y^{\prime}, z^{\prime}$ is used with $x^{\prime}$ being the wall tangential direction, $y^{\prime}$ being the wall normal direction, and $z^{\prime}$ pointing into the streamwise direction. Details of the post-processing to compute the orientation in this coordinate system is described in Appendix A. The orientation of the fibres in the tube is based on its inclination of the fibre coordinate system x", $y^{\prime \prime}, z^{\prime \prime}$ to the wall based reference coordinate system. The latter is expressed in polar coordinates, in which the azimuthal angle $\Theta$, and the polar angle $\varphi$ represent the orientation with respect to the stream wise direction. Figure 13 presents the orientation distribution of the fibres evaluated over the last $20 \mathrm{dtu}$. Note that $\varphi= \pm \pi / 2$ indicates a fibre orientation into the streamwise direction.

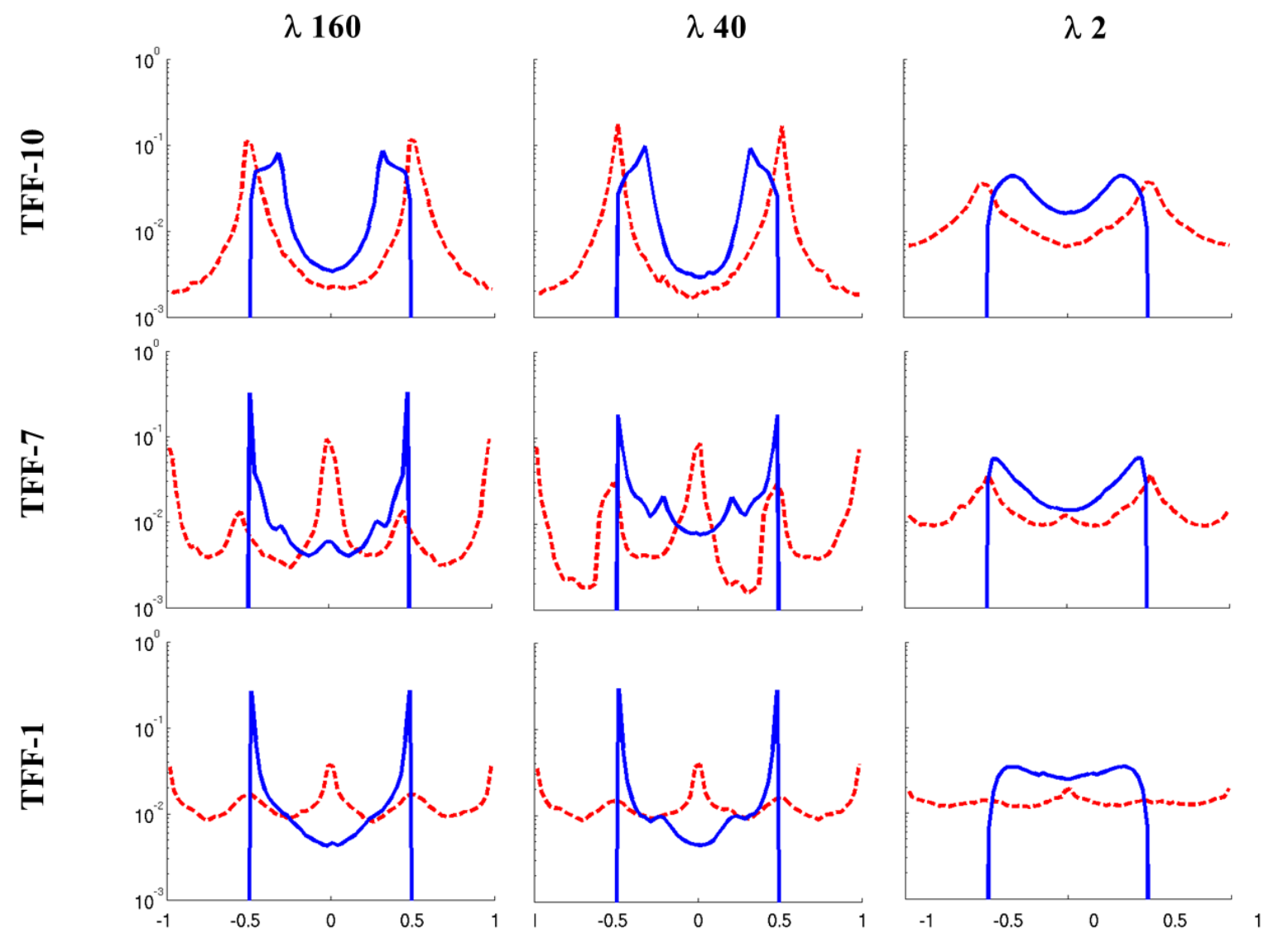

Figure 13: Fibre orientation in dependence on $\lambda$ for cases TFF-10, TFF-7, and TFF-1. Azimuthal angle $\Theta$ is drawn in red (- - ) and polar angle $\varphi$ is drawn in blue (----). The angles are given in rad with respect to $\pi$. The relative number per orientation is presented in logarithmic scale on the $y$-axis. 
We found, that $\lambda=160$ and $\lambda=40$ fibres were mostly aligned into flow direction, and hence are oriented normal to the direction of gravity. Thus, the resulting resistance to settling is high. In the range of settings, we found laminar steady or oscillatory flow. Literature reports alignment of fibres into the flow direction for laminar shear flow (Jianzhong et al., 2004). This was also found for the toroidal flow situation considered in our work. $\lambda=2$ fibres showed a preferred orientation into the flow direction, however to lower extent. For $\lambda=2$ fibres in TFF1 the orientation was found to be largely random. This is due to the short flipping time of fibres (i.e., every $0.6 \mathrm{dtu}$, Table 8) and consequently their higher flipping frequency.

\subsubsection{Residence Time of Simulated Fibres}

The (dimensionless) RT distribution of simulated fibres was calculated based on the fibre propagation compared to the fluid propagation. For TFF-10, and TFF-7 the last $2 / 3$, and for TFF-1 the total simulated time was considered for evaluation of the RT. Thus, the RT distribution reported below is an extrapolation based on an averaged fibre behavior.

Figure 14 presents the RT distribution up to $\tau=2$. However, it must be noted that not all fibres were collected in that time period. Consequently, only the amount of fibres with $\tau \leq 2$ is stated in Table 9.

Table 9: Relative number of fibres with $\tau \leq 2$ and $\tau$ of the fastest fibre per fibre class is reported.

\begin{tabular}{ccccccc}
\hline Fibre Type & \multicolumn{3}{c}{ Recovered Fibres $\tau \leq 2$} & \multicolumn{3}{c}{$\tau$ of fastest Fibres } \\
& TFF-10 & TFF-7 & TFF-1 & TFF-10 & TFF-7 & TFF-1 \\
\hline$\lambda 160$ & 0.343 & 0.414 & 1.000 & 0.752 & 0.852 & 0.844 \\
$\lambda 40$ & 0.585 & 0.434 & 0.987 & 0.752 & 0.855 & 0.860 \\
$\lambda 2$ & 0.995 & 0.993 & 0.993 & 0.756 & 0.853 & 0.861 \\
\hline
\end{tabular}

Only a small fraction of $\lambda=160$ and $\lambda=40$ fibres had a $\tau \leq 2$ for cases TFF-10, and TFF-7. For these situations we found that fibres accumulate in almost stagnant zones located at the inner wall (see the fibre positions in Figure 9 and Figure 10). Due to the fact that more $\lambda=40$ fibres settled to the lower half of the tube, and thus more fibres would accumulate in the stagnant zone, the amount of $\lambda=40$ fibres was smaller compared to $\lambda=160$ fibres. $\lambda=2$ fibres were 
nearly fully recovered and they showed a narrow RT distribution. It is worth to note that for low curvatures (TFF-10), more fibres appeared earlier compared to a situation with an increased curvature (TFF-7). This is consistent with findings from Palazoglu and Sandeep (Palazoglu and Sandeep, 2004), who found a decrease of the non-dimensional RT $\tau$ for nearly buoyant particles from 0.92 to 0.88 when decreasing the curvature from 0.143 to 0.077 . Nearly no difference in the RT $\tau$ was found for TFF-1. Here, all fibres were well mixed in the cross section and did not reside longer at areas of low axial velocity. Hence in the simulation of single fibres in the TFF, no separation occurs at higher $R e$.

TFF-10

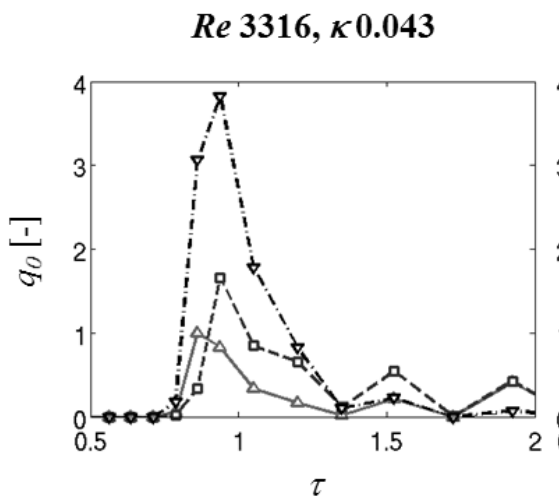

TFF-7

$\operatorname{Re} 3316, \kappa 0.1$

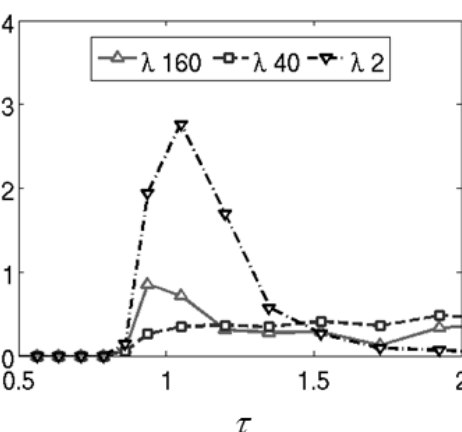

TFF-1

$\operatorname{Re} 6926, \kappa 0.1$

Figure 14: Residence time distribution of $\lambda 160$ (---), $\lambda 40$ (- - ), and $\lambda 2$ (-.-.) fibres. The frequency per time unit is given as density distribution based on the fibre number and class size $q_{0}$.

In Figure 14 we see the effect caused by the rapid settling behavior of $\lambda 40$ fibres towards the

LH. $\lambda=160$ fibres appear first, and the effect was more pronounced for a higher degree of separation (TFF-7 to TFF-10). For single fibres suspended in fluid, the TFF separates the longer fibres $(\lambda=160)$ from the shorter $(\lambda=40)$ at relative long time scales $(\tau>2)$. Smallest fibres $(\lambda$ $=2$ ) were found with the first fraction of fibres. 


\section{Summary and Discussion}

Previous speculations about the mechanism causing size based fibre separation in a TFF were based on (i) observations of separation in suspension slug flow (Olgard, 1970), and (ii) arguments using the lateral migration towards the wall and interaction with the fluid (Laitinen et al., 2011). However, for fluid flow in coiled tubes, additional motion arises in the cross section (Dean, 1928, 1927; Di Piazza and Ciofalo, 2011), which was previously not considered. Thus, the flow profile differs strongly from tube flow (Jianzhong et al., 2004) or slug flow (Talimi et al., 2012). Due to secondary motion fibres are mixed in the cross section of the tube. The ratio of the fibres' sedimentation speed and that of the fluid's secondary motion leads to a separation of fibres according to their size. The separation is highly dependent on the curvature $\kappa$ of the tube, the Re number, particle density and shape. Figure 15 summarizes some key findings from our simulations: long fibres (colored turquoise) accumulate at the inner wall in the LH. The velocity profiles indicate a reduction of the axial velocity near $\varphi=\pi$ (the velocity profile is extruded according to the streamwise velocity component).

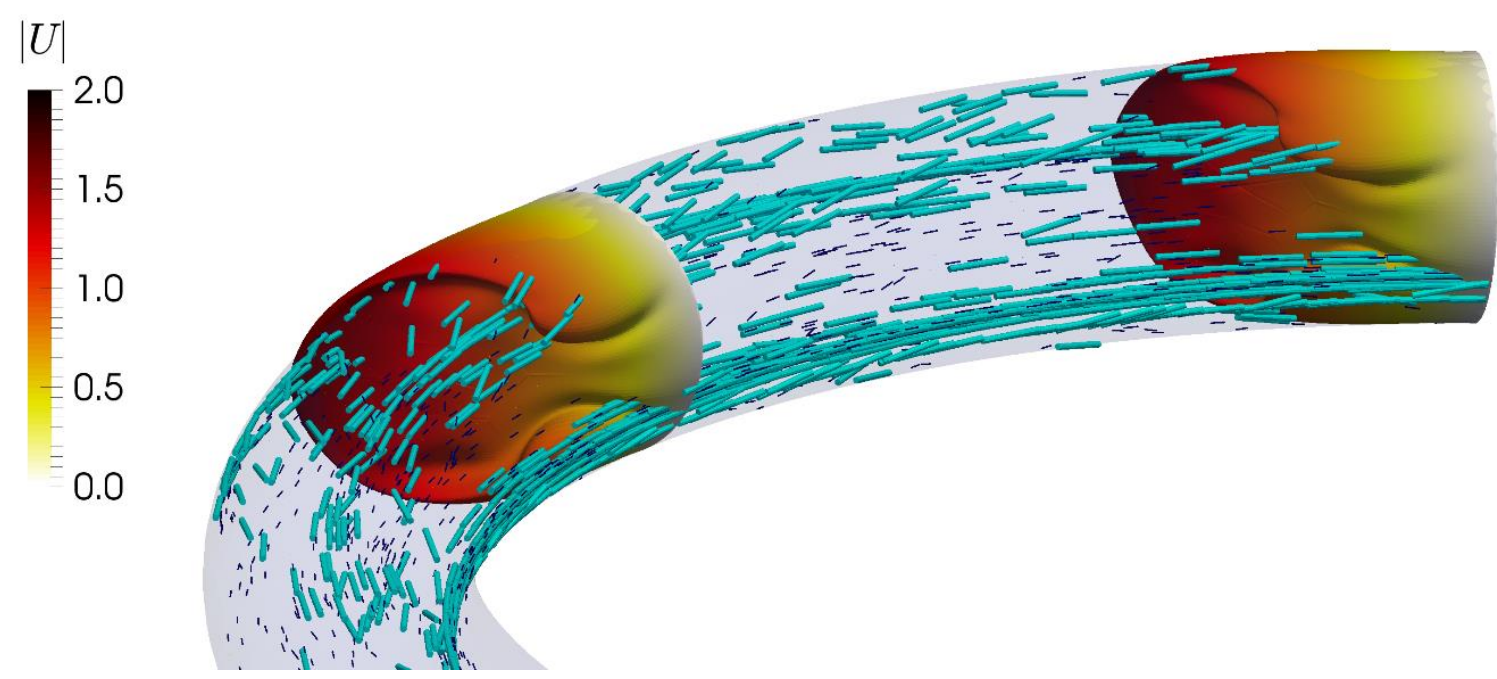

Figure 15: Snapshot of two-phase fibre flow in a toroidal tube (TFF-7, $\operatorname{Re} 3316, \kappa 0.1)$. Fluid flow profiles in two planes are extruded and colored by the magnitude of the streamwise component. $\lambda=160$ fibres are presented in turquoise, $\lambda=\mathbf{4 0}$ are presented in black.

Our experimental results (i.e., case CF-0.3-3316) are in qualitative good agreement with the corresponding simulations (i.e., TFF-10). In the experiment $0.3 \mathrm{~mm}$ fibres were first found between RT 0.76 to 0.77 for a fibre concentration of $0.3 \%$. Fibres were measured gravimetrically which gives a rather low-resolution when detecting single or small amounts of 
fibres. In the simulation we found the first fibres of $\lambda=40$, which would be $1 \mathrm{~mm}$ in the experiment at RT 0.752 . For a suspension of $0.3 \mathrm{~mm}$ long fibres at $0.1 \%$ concentration we found that more fibres could be recovered in the same time for higher Re number (see Table 5). Also, we showed by simulation that for higher $R e$, the fibres' sedimentation speed played a minor role. Thus, fewer fibres accumulated near the wall due to sedimentation. In summary, RT distribution was narrower and fewer fibres were retained in the TFF.

Simulation results differed from experimental results for CF-4-3316. In our simulation we find first an appearance of long fibres $(\lambda=160)$ at the same time as shorter fibres $(\lambda=40$, and $\lambda=2)$. Only a smaller fraction of $\lambda=160$ fibres could be recovered and they were largely retained in the TFF. In the experiment we found long fibres $(4 \mathrm{~mm})$ to leave the tube first: more than $80 \%$ of these fibres could be recovered for $\tau \leq 1$. The key difference was that most likely fibre flocculation occurred in the experiment, which was not represented in the simulation. Assuming, that fluid in the floc was dragged with the fibre network, a floc presents a large body with a density close to that of the ambient fluid. This effect is enhanced since we find that fibres accumulate at distinct orbits, and thus concentrate. This leads to an increase of the local crowding number $N_{C W}$. Ajersch (Ajersch, 1997) speculated, that for cellulose pulp suspensions that form flocs, fines (those are fibres smaller than $76 \mu \mathrm{m}$ ) accumulate in the inter-floc suspension. This would mean that moderate flocculation has a positive effect on fibre-fines separation, and that there exists an optimal fibre concentration at which the separation is most efficient. Our work showed a degradation of separation efficiency in case a bi-disperse fibre suspension was used (see Figure 4). Specifically, we could show that a relative increase of long fibres led to a decrease of the difference between the RT $\tau$ of fibres and fines. In the experiments with sulfite pulp, we found a lower residence time for all fibre classes when increasing the fibre concentration. Apparently, fibre-fluid interactions only have a weak effect. Increasing the $R e$ number slightly decreased the RT. At increased Re number, shear rate and the secondary motion are larger. We hypothesize, that these factors increase the rate of fibres that are washed from the floc, and thus move more freely through the TFF. This is in analogy to fibre suspension pipe flow. There, the turbulence level in the water annulus near the wall increases, ultimately leading to less fibres in the core and thus to a higher degree of individual suspended fibres (Derakhshandeh et al., 2011). We note in passing that an application of coiled tube flow is the generation of dense flocs (Carissimi and Rubio, 2015, 2005), indicating the importance of floc formation in these flows. 


\section{Conclusion}

We performed CFD-DEM simulations to study the motion of rigid fibres in coiled tubes, and thus explain the separation behavior found in literature, as well as in our own experiments. By comparing our simulation and experimental data, we find that the separation of fibres according to their length is not due to fibre-fluid interaction as previously believed. In contrast, it appears that separation is caused by fibre-fibre interactions, and the tendency of fibres to form coherent networks. The chance of separation is based on the chance of individual fibres to be captured in a fibre network. Thus, the fibre concentration and the ratio of long fibres in the mixture have a profound effect on the separation performance. Sample preparation for analytic purposes therefore should be performed with care. This fact is also obvious from the correlation derived by Laitinen (Laitinen et al., 2011), which only fits certain experimental settings.

We found that elongated particles with a density ratio of 1.3 (typically for cellulose fibres) move along complicated orbits in the cross section of the coiled tube for dilute suspension flow. The orbiting behavior depends on the particle settling velocity, and the intensity of the secondary motion. Our key finding is that long (and hence comparably heavy) fibres remain in the upper half of the torus, and are confined to a narrow orbit. In contrast, intermediate-length fibres settle to the lower half of the torus, while small fibres are well dispersed over the cross section. This surprising finding is similar to the recent work of Wang et.al. (Wang et al., 2014), which observed a spontaneous concentration of (spherical) particles in a stirred tank under similar flow conditions (i.e., small density ratios and moderate Reynolds numbers). This highlights that inertial effects in dilute fluid-particle suspensions, as well as moderate sedimentation speeds, can cause particles to spontaneously accumulate at certain positions in the flow.

We again stress that fibres of intermediate size rapidly settle to the lower half of the torus. This is also true for the longest fibres studied, however, these fibres surprisingly migrate slower in the vertical direction. Once arrived at the bottom wall, fibres are pushed towards the inner wall, where we find low axial velocities. This leads to a significant retardation of these particles in the tube, which is in qualitative agreement with experimental results. 
Manuscript: Mechanistic Understanding of Size-Based Fibre Separation in Coiled Tubes

In summary our findings highlight the importance of (i) design details such as the curvature $\kappa$, and (ii) operation conditions such as the Re number (or flow rate) on the residence time of fibres as a function of their size. While a large Re number yields a rather narrow residence time distribution, a larger curvature $\kappa$ can lead to a significant retardation of fibre-shaped particles, long residence times, as well as a wide residence time distribution.

In our simulations fibre flexibility was not accounted for (Andrić et al., 2014), and also fibrefibre interactions were neglected (Schmid and Klingenberg, 2000). Flexible fibres appear in different shapes and thus the hydrodynamic behavior for otherwise similar length and aspect ratio changes. Including fibre flexibility and fibre-fibre interaction into simulations would allow us to predict the effect of fibre flocculation. Hence, we expect a higher fidelity of future predictions, and possibly a quantitative agreement with experimental findings can be obtained. Unfortunately, the simulation cost is expected to increase significantly, for which reason we have addressed such investigations to future work. 
Manuscript: Mechanistic Understanding of Size-Based Fibre Separation in Coiled Tubes

\section{Acknowledgements}

We gratefully acknowledge the assistance of our students' employees Thomas Treschmitzer, and Yvonne Holzbauer, and our high-school intern Birgit Krainer with the laboratory experiments.

The authors acknowledge the industrial partners Sappi Gratkorn, Zellstoff Pöls AG, Norske Skog Bruck, and Mondi Frantschach, the Austrian Research Promotion Agency (FFG), COMET, BMVIT, BMWFJ, the Country of Styria, and Carinthias for their financial support of the K-project FLIPPR ${ }^{\circ}$.

We also gratefully acknowledge support from NAWI Graz by providing access to dcluster.tugraz.at.

LIGGGHTS $^{\circledR}$, and CFDEM ${ }^{\circledR}$ are registered trademarks of DCS Computing GmbH, the producer of the $\mathrm{CFDEM}^{\circledR}$ coupling software. This offering is not approved or endorsed by DCS Computing GmbH, the producer of the LIGGGHTS ${ }^{\circledR}$ and CFDEM $^{\circledR}$ coupling software and owner of the LIGGGHTS ${ }^{\circledR}$ and CFDEM $^{\circledR}$ trademarks. 


\section{Literature}

Ajersch, M., 1997. Mechanisms of Pulp Loss in Flotation Deinking. McMaster University.

Andrić, J., Lindström, S.B., Sasic, S., Nilsson, H., 2014. Rheological properties of dilute suspensions of rigid and flexible fibers. J. Nonnewton. Fluid Mech. 212, 36-46. doi:10.1016/j.jnnfm.2014.08.002

Bennington, C.P.J., Kerekes, R.J., Grace, J.R., 1990. The yield stress of fibre suspensions. Can. J. Chem. Eng. 68, 748-757. doi:10.1002/cjce.5450680503

Carissimi, E., Rubio, J., 2015. Polymer-bridging flocculation performance using turbulent pipe flow. Miner. Eng. 70, 20-25. doi:10.1016/j.mineng.2014.08.019

Carissimi, E., Rubio, J., 2005. The flocs generator reactor-FGR: a new basis for flocculation and solid-liquid separation. Int. J. Miner. Process. 75, 237-247. doi:10.1016/j.minpro.2004.08.021

Celzard, a., Fierro, V., Kerekes, R., 2009. Flocculation of cellulose fibres: New comparison of crowding factor with percolation and effective-medium theories. Cellulose 16, 983-987. doi:10.1007/s10570-009-9314-0

Chen, B., Tatsumi, D., Matsumoto, T., 2003. Fiber Orientation and Flow Properties of Pulp Fiber Suspensions under Shear Flow Conditions. Sen'I Gakkaishi 59, 471-478.

Ciofalo, M., Di Liberto, M., Marotta, G., 2014. On the influence of curvature and torsion on turbulence in helically coiled pipes. J. Phys. Conf. Ser. 501, 012025. doi:10.1088/1742$6596 / 501 / 1 / 012025$

Cox, R.G., 1971. The motion of long slender bodies in a viscous fluid. Part 2. Shear flow. J. Fluid Mech. 45, 625. doi:10.1017/S0022112071000259

Cui, H., Grace, J.R., 2007. Flow of pulp fibre suspension and slurries: A review. Int. J. Multiph. Flow 33, 921-934. doi:10.1016/j.ijmultiphaseflow.2007.03.004

Dean, W.R., 1928. LXXII. The stream-line motion of fluid in a curved pipe (Second paper). London, Edinburgh, Dublin Philos. Mag. J. Sci. 5, 673-695. doi:10.1080/14786440408564513

Dean, W.R., 1927. XVI. Note on the motion of fluid in a curved pipe. London, Edinburgh, Dublin Philos. Mag. J. Sci. 4, 208-223. doi:10.1080/14786440708564324

Derakhshandeh, B., Kerekes, R.J., Hatzikiriakos, S.G., Bennington, C.P.J., 2011. Rheology of pulp fibre suspensions: A critical review. Chem. Eng. Sci. 66, 3460-3470. doi:10.1016/j.ces.2011.04.017

Di Carlo, D., 2009. Inertial microfluidics. Lab Chip 9, 3038. doi:10.1039/b912547g

Di Liberto, M., Di Piazza, I., Ciofalo, M., 2013. Turbulence structure and budgets in curved pipes. Comput. Fluids 88, 452-472. doi:10.1016/j.compfluid.2013.09.028 
Di Piazza, I., Ciofalo, M., 2011. Transition to turbulence in toroidal pipes. J. Fluid Mech. 687, 72-117. doi:10.1017/jfm.2011.321

Di Piazza, I., Ciofalo, M., 2010. Numerical prediction of turbulent flow and heat transfer in helically coiled pipes. Int. J. Therm. Sci. 49, 653-663. doi:10.1016/j.ijthermalsci.2009.10.001

Do-Quang, M., Amberg, G., Brethouwer, G., Johansson, a. V., 2014. Simulation of finite-size fibers in turbulent channel flows. Phys. Rev. E - Stat. Nonlinear, Soft Matter Phys. 89, 115. doi:10.1103/PhysRevE.89.013006

Eder, R.J.P., Radl, S., Schmitt, E., Innerhofer, S., Maier, M., Gruber-Woelfler, H., Khinast, J.G., 2010. Continuously Seeded, Continuously Operated Tubular Crystallizer for the Production of Active Pharmaceutical Ingredients. Cryst. Growth Des. 10, 2247-2257. doi:10.1021/cg9015788

Goniva, C., Kloss, C., Deen, N.G., Kuipers, J.A.M., Pirker, S., 2012. Influence of rolling friction on single spout fluidized bed simulation. Particuology 10, 582-591. doi:10.1016/j.partic.2012.05.002

Hourani, M.J., 1988. Fiber flocculation in pulp suspension flow, Part 2: Experimental results. TAPPI J. 71, 186-189.

Hüttl, T.., Friedrich, R., 2001. Direct numerical simulation of turbulent flows in curved and helically coiled pipes. Comput. Fluids 30, 591-605. doi:10.1016/S0045-7930(01)00008-1

Hüttl, T.J., Friedrich, R., 2000. Influence of curvature and torsion on turbulent flow in helically coiled pipes. Int. J. Heat Fluid Flow 21, 345-353. doi:10.1016/S0142-727X(00)00019-9

Ito, H., 1959. Friction Factors for Turbulent Flow in Curved Pipes. Trans. ASME J. Basic Engng. 81, 123-134.

Jagiello, L.A., 2013. Implementation of a Tube Flow Fractionator. Graz University of Technology.

Jeffery, G.B., 1922. The Motion of Ellipsoidal Particles Immersed in a Viscous Fluid. Proc. R. Soc. A Math. Phys. Eng. Sci. 102, 161-179. doi:10.1098/rspa.1922.0078

Jianzhong, L., Weifeng, Z., Zhaosheng, Y., 2004. Numerical research on the orientation distribution of fibers immersed in laminar and turbulent pipe flows. J. Aerosol Sci. 35, 6382. doi:10.1016/S0021-8502(03)00388-4

Johansson, H., Olgard, G., Jernqvist, A., 1970. Radial particle migration in plug flow - a method for solid-liquid separation and fractionation. Chem. Eng. Sci. 25, 365-372. doi:10.1016/0009-2509(70)80035-5

Kerekes, 2006. Rheology of fibre suspensions in papermaking: An overview of recent research. Nord. Pulp Pap. Res. J. 21, 598-612. doi:10.3183/NPPRJ-2006-21-05-p598-612

Kim, S., Karrila, S.J., 2005. Microhydrodynamics: Principles and Selected Applications, Butterworth - Heinemann series in chemical engineering. Dover Publications. 
Körkkö, M., Laitinen, O., Vahlroos, S., Ämmälä, A., Niinimäki, J., 2008. Components Removal in Flotation Deinking 17, 15-22.

Koutsky, J.A., Adler, R.J., 1964. Minimisation of axial dispersion by use of secondary flow in helical tubes. Can. J. Chem. Eng. 42, 239-246. doi:10.1002/cjce.5450420602

Krochak, P.J., Olson, J. a., Martinez, D.M., 2009. Fiber suspension flow in a tapered channel: The effect of flow/fiber coupling. Int. J. Multiph. Flow 35, 676-688. doi:10.1016/j.ijmultiphaseflow.2009.03.005

Krogerus, B., Fagerholm, K., Löytynoja, L., 2003. Analytical fractionation of pulps by tube flow. Pap. ja Puu 85, 209-213.

Kühnen, J., Holzner, M., Hof, B., Kuhlmann, H.C., 2013. Experimental investigation of transitional flow in a toroidal pipe. J. Fluid Mech. 738, 463-491. doi:10.1017/jfm.2013.603

Kvick, M., 2014. Transitional and turbulent fibre suspension flows. KTH Royal Institute of Technology.

Laitinen, O., Kemppainen, K., Stoor, T., Niinimäki, J., 2011. Fractionation of pulp and paper particles selectively by size. BioResources 6, 672-685.

Laitinen, O., Körkkö, M., Niinimäki, J., 2008. The Effects of Aging in Different Raw Material Furnishes. Progess Pap. Recycl. 18, 9-15.

Laitinen, O., Löytynoja, L., Niinimäki, J., 2006. Tube flow fractionator - A simple method for laboratory fractionation. Pap. ja Puu 88, 351-355.

Lin, J., Shi, X., Yu, Z., 2003. The motion of fibers in an evolving mixing layer. Int. J. Multiph. Flow 29, 1355-1372. doi:10.1016/S0301-9322(03)00086-7

Lindström, S.B., Uesaka, T., 2007. Simulation of the motion of flexible fibers in viscous fluid flow. Phys. Fluids 19. doi:10.1063/1.2778937

Lu, G., Third, J.R., Müller, C.R., 2015. Discrete element models for non-spherical particle systems: From theoretical developments to applications. Chem. Eng. Sci. 127, 425-465. doi:10.1016/j.ces.2014.11.050

Lundell, F., Carlsson, A., 2010. Heavy ellipsoids in creeping shear flow: Transitions of the particle rotation rate and orbit shape. Phys. Rev. E 81, 016323. doi:10.1103/PhysRevE.81.016323

Lundell, F., Söderberg, L.D., Alfredsson, P.H., 2011. Fluid Mechanics of Papermaking. Annu. Rev. Fluid Mech. 43, 195-217. doi:10.1146/annurev-fluid-122109-160700

Marchioli, C., Fantoni, M., Soldati, A., 2010. Orientation, distribution, and deposition of elongated, inertial fibers in turbulent channel flow. Phys. Fluids 22, 1-14. doi:10.1063/1.3328874

Marchioli, C., Soldati, A., 2013. Rotation statistics of fibers in wall shear turbulence. Acta Mech. 224, 2311-2329. doi:10.1007/s00707-013-0933-z 
Martel, J.M., Toner, M., 2013. Particle Focusing in Curved Microfluidic Channels. Sci. Rep. 3, 1-8. doi:10.1038/srep03340

Mortensen, P.H., Andersson, H.I., Gillissen, J.J.J., Boersma, B.J., 2008a. On the orientation of ellipsoidal particles in a turbulent shear flow. Int. J. Multiph. Flow 34, 678-683. doi:10.1016/j.ijmultiphaseflow.2007.12.007

Mortensen, P.H., Andersson, H.I., Gillissen, J.J.J., Boersma, B.J., 2008b. Dynamics of prolate ellipsoidal particles in a turbulent channel flow. Phys. Fluids 20, 093302. doi:10.1063/1.2975209

Naphon, P., Wongwises, S., 2006. A review of flow and heat transfer characteristics in curved tubes. Renew. Sustain. Energy Rev. 10, 463-490. doi:10.1016/j.rser.2004.09.014

Noorani, A., El Khoury, G.K., Schlatter, P., 2013. Evolution of turbulence characteristics from straight to curved pipes. Int. J. Heat Fluid Flow 41, 16-26. doi:10.1016/j.ijheatfluidflow.2013.03.005

Olgard, G., 1970. Fractionation of Fiber Suspensions by Liquid Column Flow. TAPPI J. 53, 1240-1246.

Palazoglu, T.K., Sandeep, K.P., 2004. Effect of tube curvature ratio on the residence time distribution of multiple particles in helical tubes. LWT - Food Sci. Technol. 37, 387-393. doi:10.1016/S0023-6438(03)00013-6

Redlinger-Pohn, J.D., König, L.M., Kloss, C., Goniva, C., Radl, S., 2016. Modelling of NonSpherical, Elongated Particles for Industrial Suspension Flow Simulation, in: Papadrakakis, M., Papadopoulos, V., Stefanou, G., Plevris, V. (Eds.), VII European Congress on Computational Methods in Applied Sciences and Engineering. Crete Island, p. 14.

Rosén, T., Lundell, F., Aidun, C.K., 2014. Effect of fluid inertia on the dynamics and scaling of neutrally buoyant particles in shear flow. J. Fluid Mech. 738, 563-590. doi:10.1017/jfm.2013.599

Sandeep, K.P., Zuritz, C. a, Puri, V.M., 1997. An Hypothesis Paper Residence Time Distribution of Particles during Two-Phase Non-Newtonian Flow in Conventional as compared with Helical Holding Tubes 62, 647-652.

Schmid, C.F., Klingenberg, D.J., 2000. Mechanical Flocculation in Flowing Fiber Suspensions. Phys. Rev. Lett. 84, 290-293. doi:10.1103/PhysRevLett.84.290

Switzer, L.H., Klingenberg, D.J., 2004. Flocculation in simulations of sheared fiber suspensions. Int. J. Multiph. Flow 30, 67-87. doi:10.1016/j.ijmultiphaseflow.2003.10.005

Talimi, V., Muzychka, Y.S., Kocabiyik, S., 2012. A review on numerical studies of slug flow hydrodynamics and heat transfer in microtubes and microchannels. Int. J. Multiph. Flow 39, 88-104. doi:10.1016/j.ijmultiphaseflow.2011.10.005

Tiwari, P., Antal, S.P., Podowski, M.Z., 2006. Three-dimensional fluid mechanics of particulate two-phase flows in U-bend and helical conduits. Phys. Fluids 18, 043304. 
Manuscript: Mechanistic Understanding of Size-Based Fibre Separation in Coiled Tubes

doi:10.1063/1.2189212

Vashisth, S., Kumar, V., Nigam, K.D.P., 2008. A Review on the Potential Applications of Curved Geometries in Process Industry. Ind. Eng. Chem. Res. 47, 3291-3337. doi:10.1021/ie701760h

Wang, S., Metcalfe, G., Stewart, R.L., Wu, J., Ohmura, N., Feng, X., Yang, C., 2014. Solidliquid separation by particle-flow-instability. Energy Environ. Sci. 7, 3982-3988. doi:10.1039/C4EE02841D

Zhao, F., Van Wachem, B.G.M., 2013. Direct numerical simulation of ellipsoidal particles in turbulent channel flow. Acta Mech. 224, 2331-2358. doi:10.1007/s00707-013-0921-3 


\section{Appendix}

\subsection{Appendix A - Simulation Post Processing}

\subsubsection{Toroidal Coordinates and Transformation of Fibres into One Cross Section}

Cartesian and toroidal coordinates are expressed with respect to Figure 6. Cartesian coordinates are first transformed into toroidal coordinates:

$$
\begin{gathered}
R=\sqrt{y^{2}+z^{2}} \\
\Theta=\arctan \left(\frac{z}{y}\right) \\
\varphi=\arctan \left(\frac{x}{R-D / 2}\right) \\
r=\sqrt{x^{2}+(R-D / 2)^{2}}
\end{gathered}
$$

Fibre properties, i.e. the fibre velocity or fibre orientation were projected into the inlet plane, $\Theta$ $=0$.

$$
\text { value }_{\text {inlet }}=\left(\begin{array}{ccc}
1 & 0 & 0 \\
0 & \cos (\Theta) & \sin (\Theta) \\
0 & -\sin (\Theta) & \cos (\Theta)
\end{array}\right) \cdot \text { value }_{\text {torus }}
$$

Following the fibres coordinate system was rotated that the y coordinate is wall normal for each individual fibre. Coordinate system is rotated by $\varphi$ :

$$
\text { value }_{\text {inlet }}=\left(\begin{array}{ccc}
\cos (\varphi) & -\sin (\varphi) & 0 \\
\sin (\varphi) & \cos (\varphi) & 0 \\
0 & 0 & 1
\end{array}\right) \cdot \text { value }_{\text {torus }}
$$


Manuscript: Mechanistic Understanding of Size-Based Fibre Separation in Coiled Tubes

The orientation of the fibre in the tube is then stated by the inclination of the wall normal coordinate system x', $y^{\prime}, z^{\prime}$ to the fibre coordinate system x", $y^{\prime \prime}, z^{\prime \prime}$. Fibre orientation is expressed by the azimuthal angle $\Theta$, and the polar angle $\varphi$. The three principal directions of the fibre, wall normal, wall tangential, and streamwise with the according angles is presented in Figure $i$.

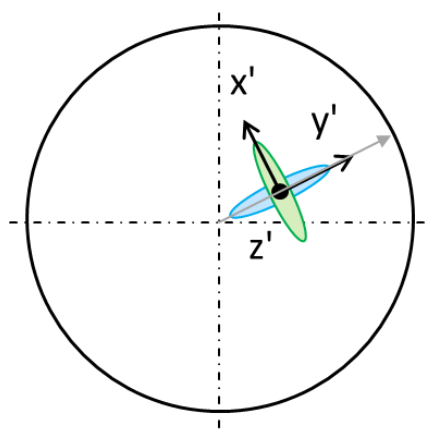

\begin{tabular}{|c|c|c|c|c|}
\cline { 2 - 5 } \multicolumn{2}{c|}{} & $\begin{array}{c}\text { Azimuthal } \\
\text { angle } \\
\Theta\end{array}$ & $\begin{array}{c}\text { Polar } \\
\text { angle } \\
\varphi\end{array}$ & $\begin{array}{c}\text { Rotation } \\
\text { axis }\end{array}$ \\
\hline \multirow{2}{*}{$\begin{array}{c}\mathrm{y} \\
\text { wall } \\
\text { normal }\end{array}$} & $\begin{array}{c}\pi / 2 \\
3 \pi / 2\end{array}$ & $\begin{array}{c} \\
\mathrm{x}\end{array}$ & $\mathrm{z}$ \\
\hline & $\begin{array}{c}\mathrm{x} \\
\text { wall } \\
\text { tangential }\end{array}$ & 0 & 0 & $\mathrm{z}$ \\
$\mathrm{z}$ & $\pi$ & $\pi$ & $\mathrm{y}$ \\
\hline & $\begin{array}{c}\mathrm{z} \\
\text { stream } \\
\text { wise }\end{array}$ & $\pi$ & $\pi / 2$ & $\mathrm{y}$ \\
\hline
\end{tabular}

Figure i: Principle directions of the fibres and according azimuthal and polar angle. 